\title{
- TID/SNA-267
}

To AEC-NASA Space Nuclear Systems Office

s131-MPM07-W187

MATERIALS PRIORITY AND MATURITY STATUS

NERVA Program, Contract SNP-1

15 February 1971 


\section{DISCLAIMER}

This report was prepared as an account of work sponsored by an agency of the United States Government. Neither the United States Government nor any agency Thereof, nor any of their employees, makes any warranty, express or implied, or assumes any legal liability or responsibility for the accuracy, completeness, or usefulness of any information, apparatus, product, or process disclosed, or represents that its use would not infringe privately owned rights. Reference herein to any specific commercial product, process, or service by trade name, trademark, manufacturer, or otherwise does not necessarily constitute or imply its endorsement, recommendation, or favoring by the United States Government or any agency thereof. The views and opinions of authors expressed herein do not necessarily state or reflect those of the United States Government or any agency thereof. 


\section{DISCLAIMER}

Portions of this document may be illegible in electronic image products. Images are produced from the best available original document. 
15 February 1971

NOTICE

This report was prepared as an account of work sponsored by the United States Government. Neither the United States nor the United States Energy Research and Development Administration, nor any of their employees, nor any of their contractors, subcontractors, or their employees, makes any warranty, express or implied, or assumes any legal liability or responsibility for the accuracy, completeness or usefulness of any information, apparatus, product or process disclosed, or represents that its use would not infringe privately owned rights.

\section{QW}

C. W. Funk, Manager

Materials and Processes

Scientific Staff Department

Aerojet Nuclear Systems Company
Classification Category UNCLASSIFIED

$\frac{c \mu=}{\text { Classifying Officer }} \frac{17 \text { reb } 71}{\text { Date }}$


MATERIAL TEST PLAN INDEX

\begin{tabular}{|c|c|c|}
\hline $\begin{array}{l}\text { TEST } \\
\text { PLAN }\end{array}$ & . & PAGE \\
\hline$M-1$ & ALLOY 718 & $1 \& 1 a$ \\
\hline$M-2$ & AISI 347 & $2 \& 2 a$ \\
\hline$M-3$ & AA $7039-T 63$ & $3 \& 3 a$ \\
\hline$M-4$ & $T I-5 A L-2.5 S N$ ELI & $4 \& 4 a$ \\
\hline$M-5$ & $A-286$ & $5 \& 5 a$ \\
\hline$M-6$ & FIBROUS GRAPHITE & $6 \& 6 a$ \\
\hline$M-7$ & AA $6061-T 6$ & $7 \& 7 a$ \\
\hline$M-9$ & $T I-6 A L-4 V$ & $9 \& 9 a$ \\
\hline$M-10$ & AISI 301 STAINLESS STEEL, $1 / 4$ HARD & 10 \\
\hline$M-11$ & FRICTION AND WEAR & 11 \\
\hline$M-12$ & HASTELLOY $X$ & $12 \& 12$ \\
\hline$M-14$ & FEURALON POLYIMIDE & $14 a$ \\
\hline$M-16$ & MARAGING STEEL (18 NI) & $16 \& 16$ \\
\hline$M-19$ & AISI $440 C$ & 19 \\
\hline$M-20$ & AA5086 & $20 \& 20$ \\
\hline$M-21$ & $7075-\mathrm{T} 73$ & $21 \& 21$ \\
\hline$M-22$ & $\mathrm{GH}_{2}$ EMBRITTLEMENT & 22 \\
\hline$M-23$ & STRESS AND RADIATION SYNERGISTIC EFFECTS & $23 a$ \\
\hline$M-29$ & AISI 310 & 29 \\
\hline$M-30$ & MULTIPHASE & $30 \& 30$ \\
\hline$M-31$ & 9310 & $31 \& 31$ \\
\hline$M-37$ & BRONZE & $37 \& 37$ \\
\hline$M-38$ & STAINLESS STEEL 22-13-5 (ARMCO) & $38 \& 38$ \\
\hline M-39 & $\mathrm{BeCu}$ & $39 \& 39$ \\
\hline$M-40$ & $\mathrm{LH}_{2}, \mathrm{LN}_{2}, \mathrm{GH}_{2}$, GHe CORRELATION & $40 a$ \\
\hline$M-44$ & $A A 2219-T 6$ & 44 \\
\hline
\end{tabular}




\begin{tabular}{|c|c|c|c|}
\hline PRIORITY & & $\begin{array}{l}\text { TEST } \\
\text { PLAN }\end{array}$ & PAGE \\
\hline 1 & $T i-5 A 1-2.5 S n$ & $M-4$ & $4 \& 4 a$ \\
\hline 2 & Gas-Radiation Correlation & $M-40$ & $40 a$ \\
\hline 3 & Stress, Radiation, Hydrogen Synergistic & $M-23$ & $23 a$ \\
\hline 4 & $\mathrm{GH}_{2}$ Embrittlement & $M-22$ & 22 \\
\hline 5 & Fibrous Graphite & $M-6$ & $6 \& 6 a$ \\
\hline 6 & 22-13-5 ARMCO SS & $M-38$ & $38 \& 38 a$ \\
\hline 7 & $7075-\mathrm{T} 73$ & $M-21$ & $21 \& 21 a$ \\
\hline 8 & 347 SS & $M-2$ & $2 \& 2 a$ \\
\hline 9 & A286 & $M-5$ & $5 \& 5 a$ \\
\hline 10 & Feuralon Polyimide & $M-14$ & $14 a$ \\
\hline 11 & $6061-T 6$ & $M-7$ & $7 \& 7 a$ \\
\hline 12 & Carburized 9310 & $M-31$ & $31 \& 31 a$ \\
\hline 13 & $\mathrm{BeCu}$ & $M-39$ & $39 \& 39 a$ \\
\hline 14 & Bronze & $M-37$ & $37 \& 37 a$ \\
\hline 15 & Friction/Wear & $M-11$ & 11 \\
\hline 16 & Hastelloy $X$ & $M-12$ & $12 \& 12 a$ \\
\hline 17 & $310 \mathrm{SS}$ & $M-29$ & 29 \\
\hline 18 & $440 \mathrm{C}$ & $M-19$ & 19 \\
\hline 19 & Maraging Stee1 & $M-16$ & $16 \& 16 a$ \\
\hline 20 & $T i-6 \mathrm{Al}-4 \mathrm{~V}$ & $M-9$ & $9 \& 9 a$ \\
\hline 21 & Multiphase & $M-30$ & $30 \& 30 a$ \\
\hline 22 & 718 & $M-1$ & $1 \& 1 a$ \\
\hline 23 & 7039-T63 & $M-3$ & $3 \& 3 a$ \\
\hline 24 & $2219-\mathrm{T} 6$ & $M-44$ & 44 \\
\hline 25 & 301 (1/4 Hard) & $M-10$ & 10 \\
\hline 26 & 5086 & $M-20$ & $20 \& 20 a$ \\
\hline
\end{tabular}


15 FEBRUARY 1971

\section{KEY TO MATERIAL PRIORITY AND MATURITY STATUS}

STATUS

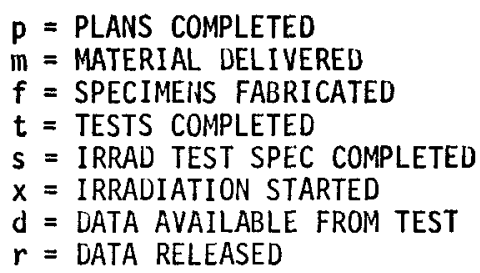

$\begin{aligned} T & =\text { TUBE } \\ W & =W E L D\end{aligned}$
DATA CATEGORIES

A

B SEE TL) 69-37

D
DECISION

(D) $=$ DESIGN

$(M)=$ MATERIAL DEVELOPMENT
ATMOSPHERE

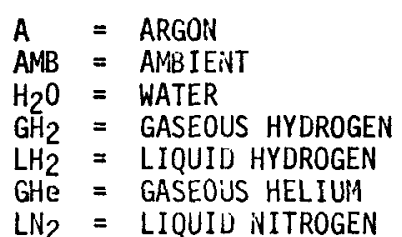

TEST FACILITY

$\begin{array}{ll}A & =\text { AEROJET } \\ B & =\text { BOEING } \\ \text { BMI } & =\text { BATTELLE MEMORIAL INSTITUTE } \\ G D & =\text { GENERAL DYNAMICS } \\ G E & =\text { GENERAL ELECTRIC } \\ \text { KSC } & =\text { KENNEUY SPACE CENTER } \\ 0 & =\text { OUTSIDE AGC (NOT CURRENTLY IUENTIFIED) } \\ \text { PBRF } & =\text { PLUMBROOK } \\ R & =\text { ROCKETDYNE } \\ \text { NBS } & =\text { NATIONAL BUREAU OF STANDARDS }\end{array}$




$$
M-1 \text { - ALLOY } 718
$$

MATERIAL PRIORITY AND MATURITY STATUS
15 FEBRUARY 1971

(INVESTIGATOR: P. P. DESSAU)

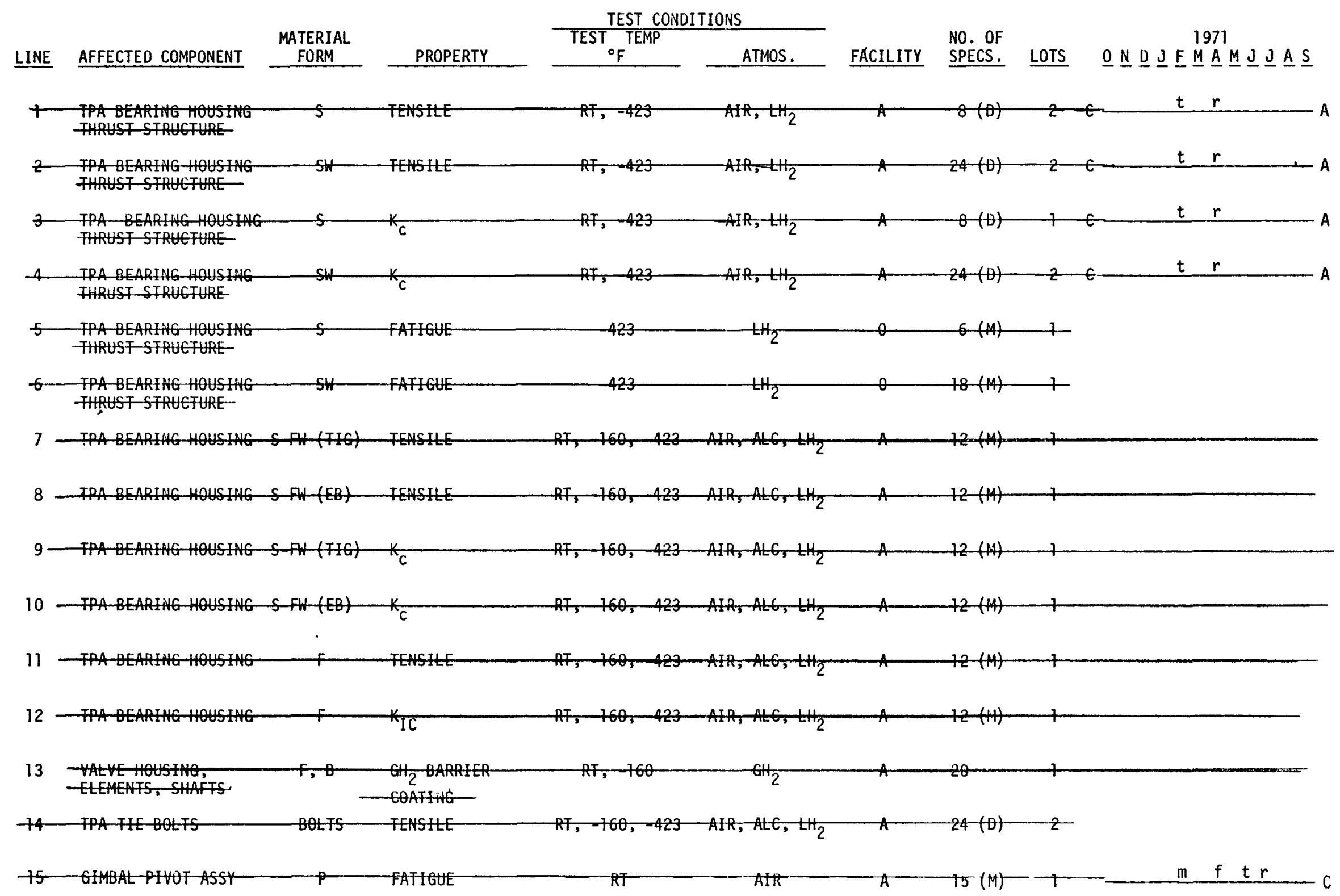


M-1 INCONEL 718

MATERIALS DATA AND MATURITY STATUS (MATERIALS IRRADIATION)

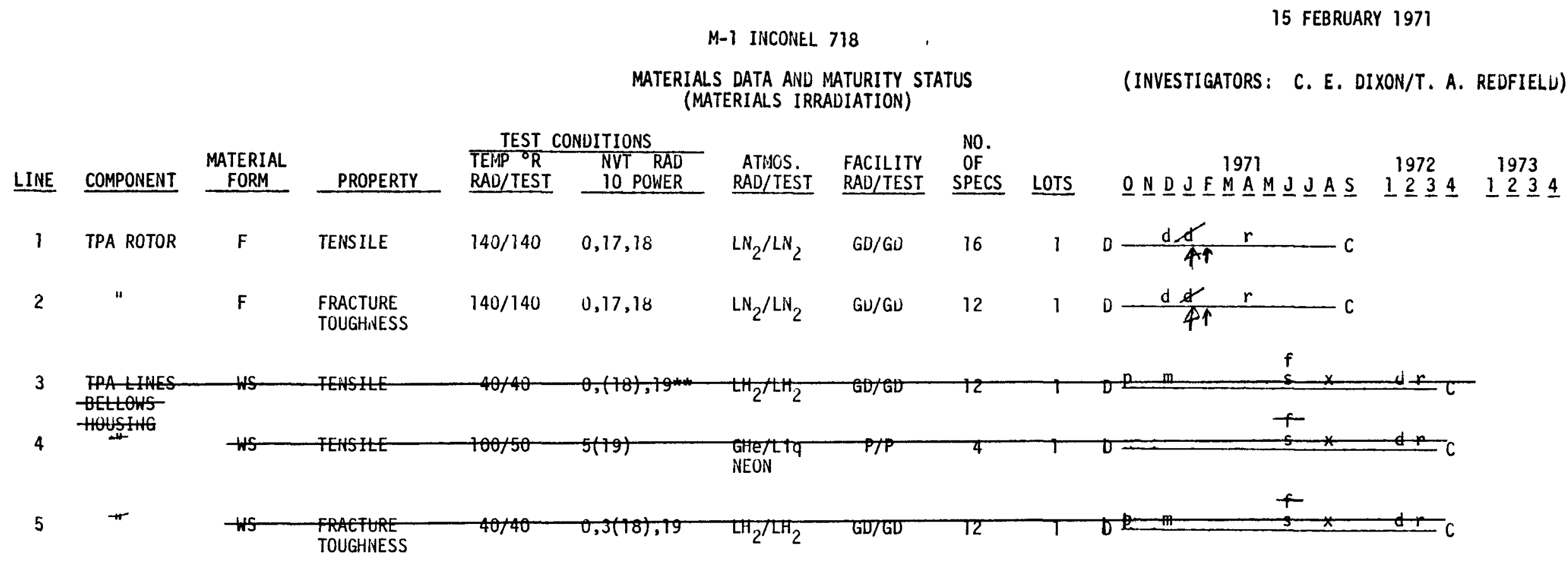



M-2 AISI 347

MATERIALS DATA AND MATURITY STATUS (MATERIALS IRRADIATION)
15 FEBRUARY 1971

(INVESTIGATORS: C. E. DIXON/T. A. REDFIELD)

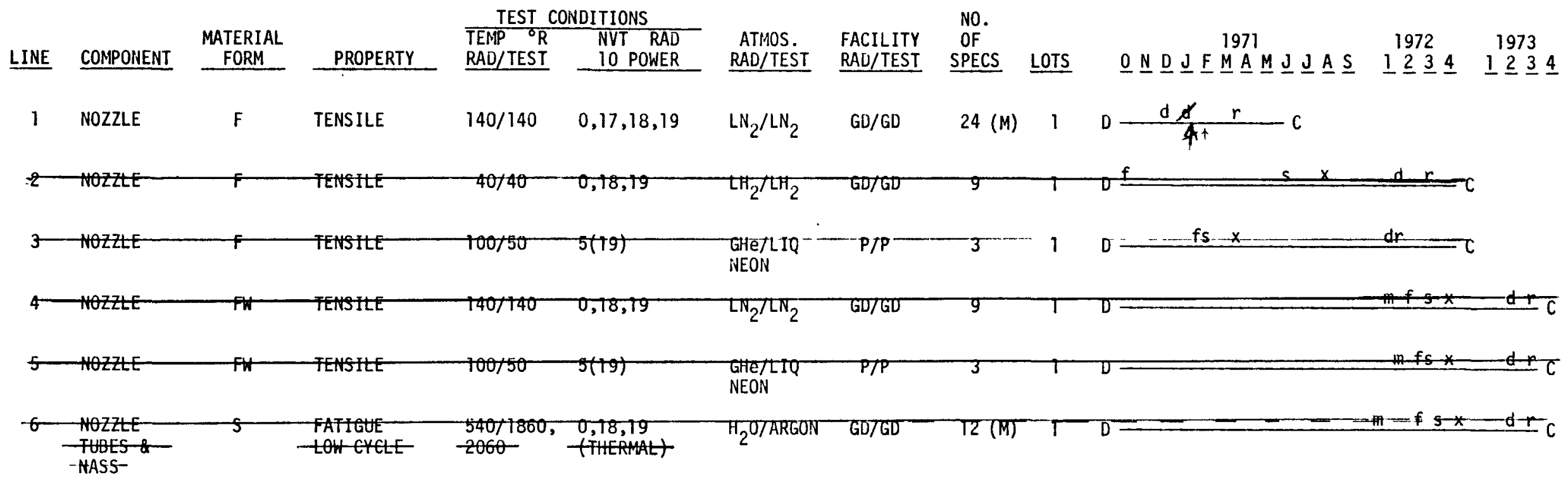


M-3 AA 7039-T63 - .

MATERIAL PRIORITY AND MATURITY STATUS
15 FEBRUARY. 1971

(INVESTIGATOR: D. J. LAMVERMEYER)

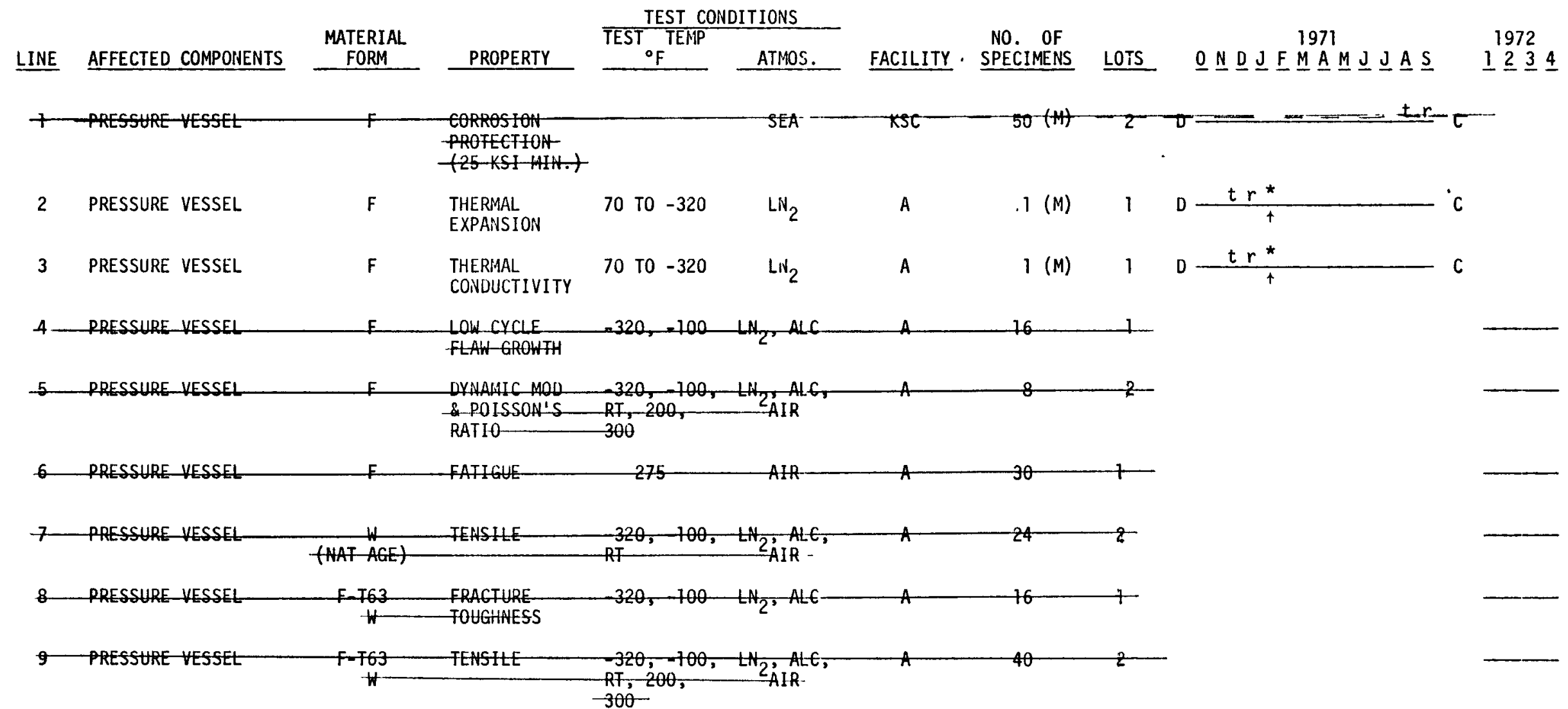

*COMPLETED 


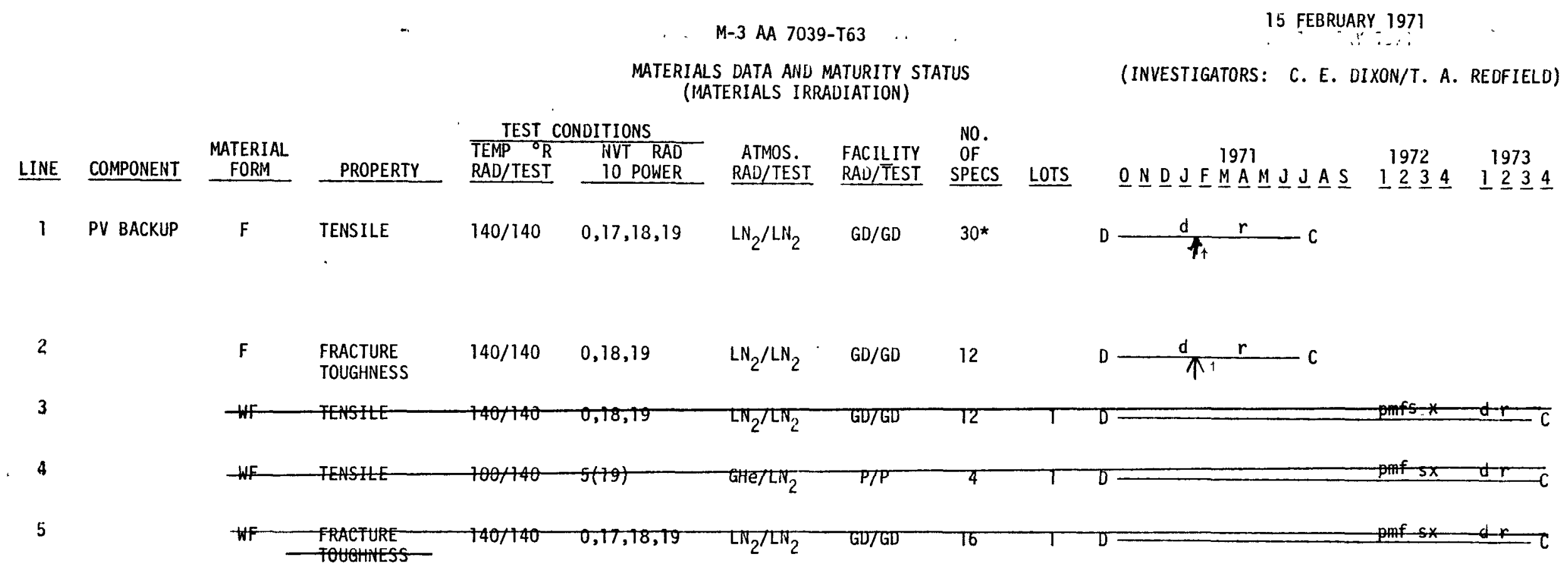

*14 specimens to be annealed. 
M-4 -. TI-5AL-2.5SN ELI

MATERIAL PRIORITY AND MATURITY STATUS
1.5 FEBRUARY : 197.1

(INVESTIGATOR: P. P. DESSAU)

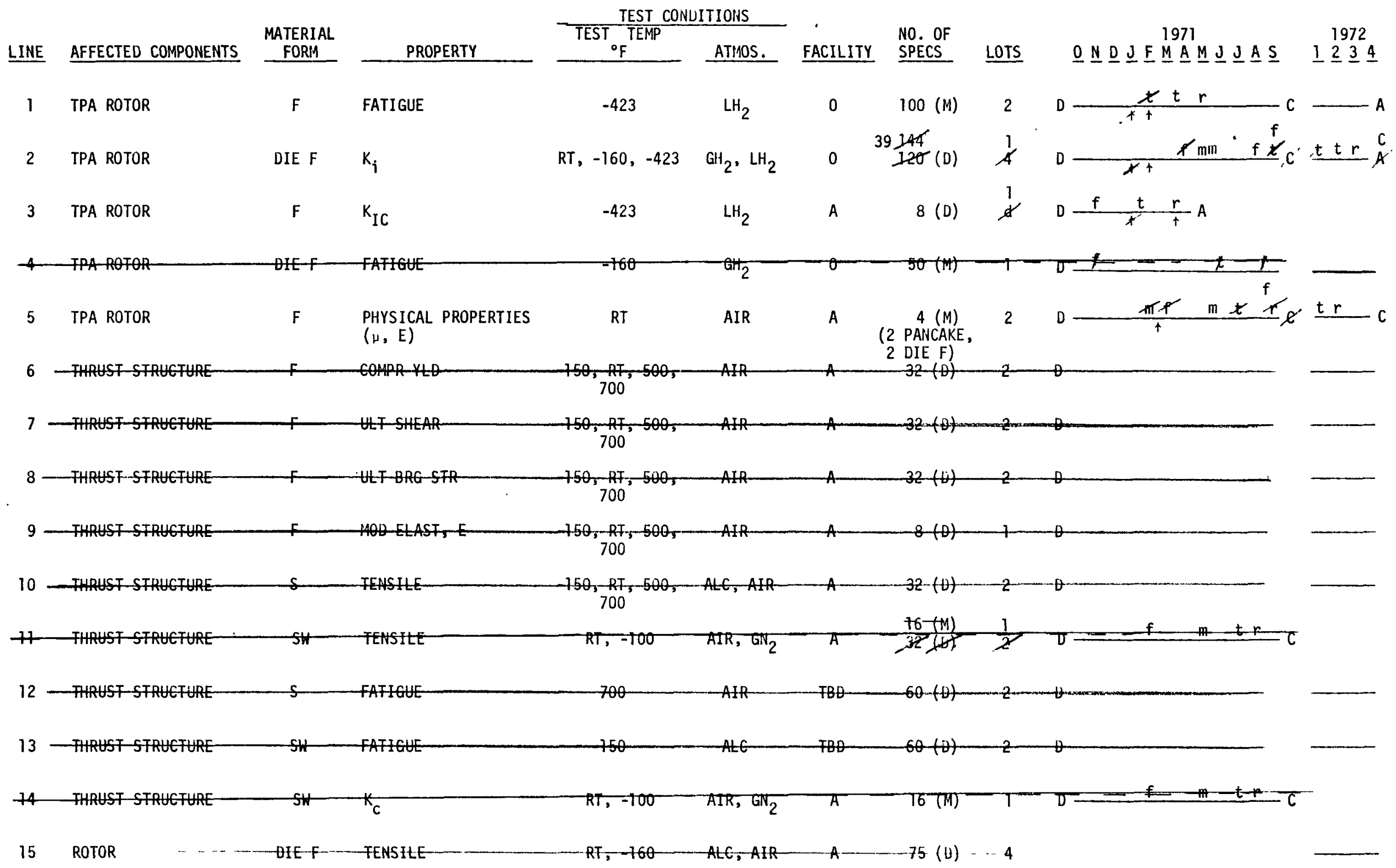


$M-4$ TI-5AL-2.5SN ELI

MATERIALS DATA AND MATURITY STATUS (MATERIALS IRRADIATION)
15 FEBRUARY 1971

(INVESTIGATORS: C. E. DIXON/T. A. REUFIELU)

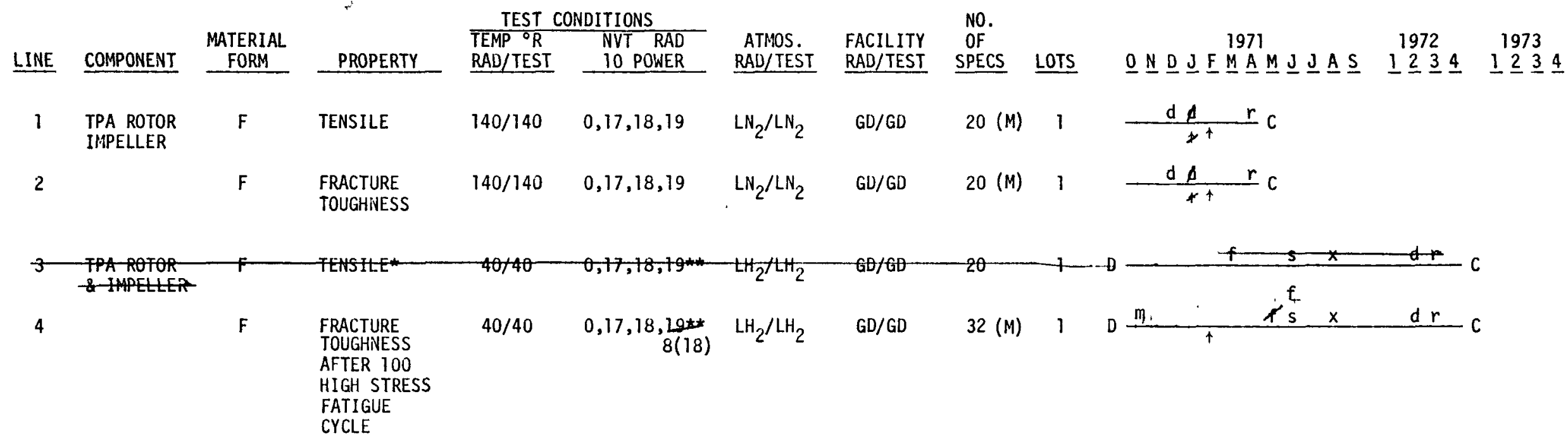

*SEE MATERIALS MEMO 70-322, 27 AUGUST 1970.

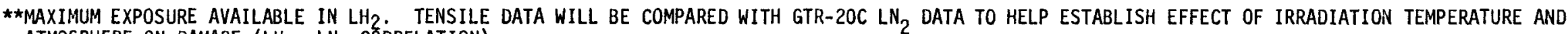
ATMOSPHERE ON DAMAGE (LH, $\mathrm{LN}_{2}$ CORRELATION). 
M-b A286 , . . , ,

MATERIAL PRIORITY AND MATURITY STATUS
15 FEBRUARY 1971

(INVESTIGATOR: P. P. DESSAU)

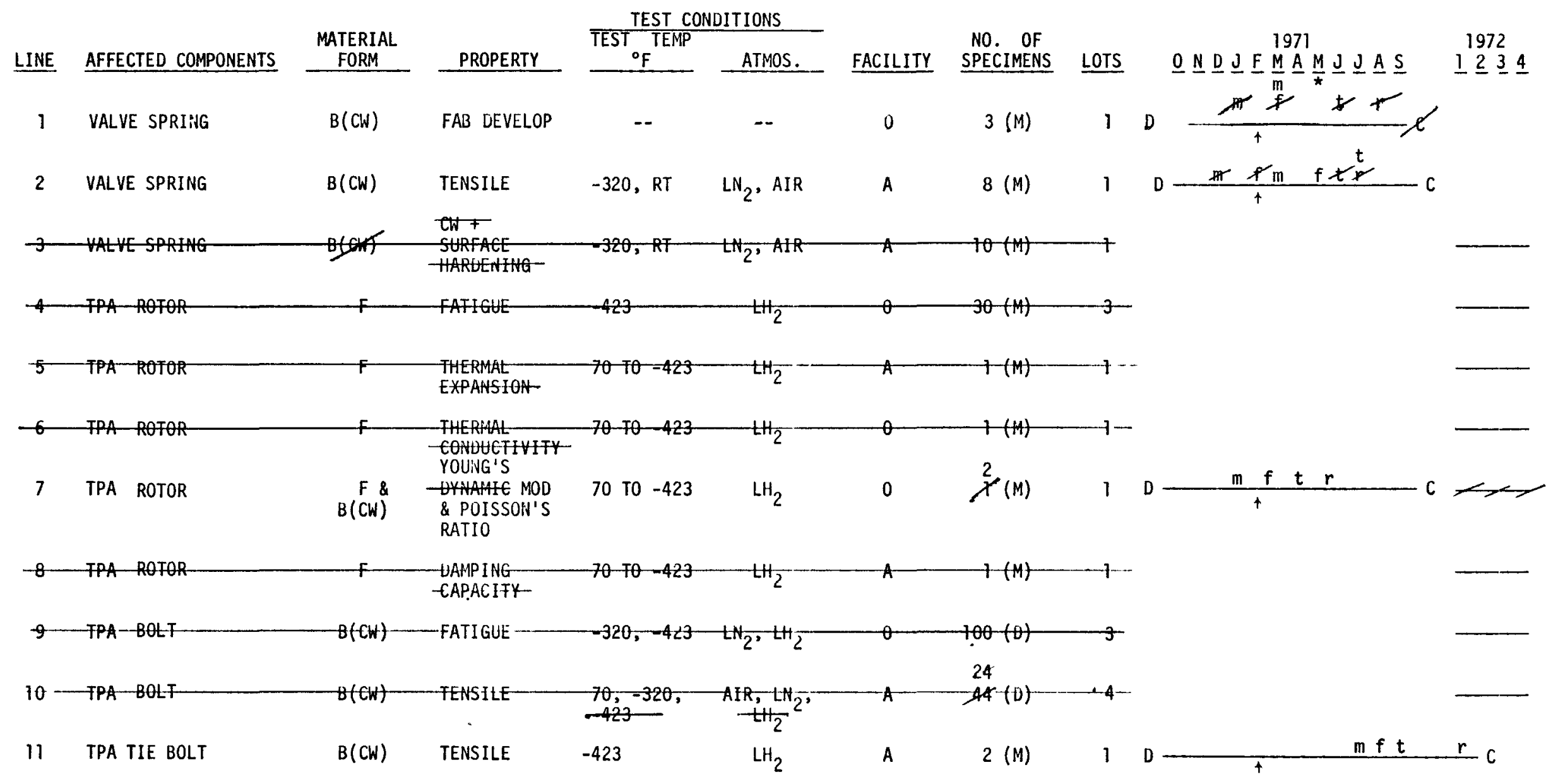

*Use material on lines 2 and 7. 
M-5 A286 $\because ;$

MATERIALS DATA AND MATURITY STATUS (MATERIALS IRRADIATION)
IS JAIUUARY 1971

(INVESTIGATORS: C. E. DIXON/T. A. REDFIELD)

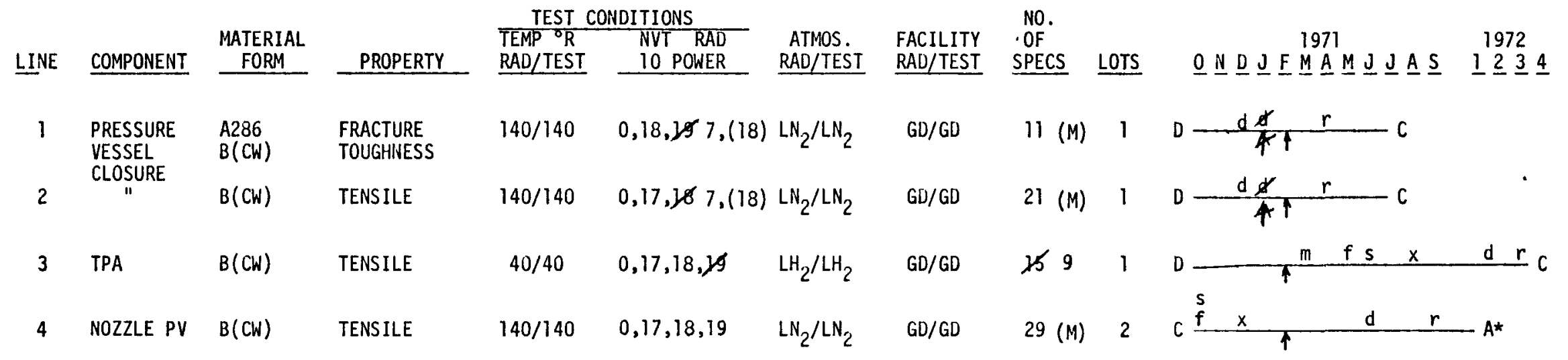

*"A" DATA FOR ELONGATION ONLY. GENERALLY NOT A DESIGN REQUIREMENT. 
M-6 - GRAPHITE COMPOSITE

MATERIAL. PRIORITY ANU MATURITY STATUS
15 FEBRUARY 1971

(INVESTIGATOR: L. M. SWOPE)

UIRECTION $\begin{aligned} & \text { TEMP } \\ & { }^{\circ} \mathrm{F}\end{aligned}$ ATMOS.

UIRECTION $\begin{aligned} & \text { TEMP } \\ & { }^{\circ} \mathrm{F}\end{aligned}$ ATMOS.

NOZZLE

EXTEISSION

11

$"$

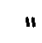

BINUER UEVELOP.

\begin{tabular}{cl} 
FORM & \multicolumn{1}{c}{ PROPERTY } \\
PLATE & BINUER UEVELOP. \\
& TENSILE \\
$"$ & COMP \\
$"$ & SHEAR \\
$"$ & MODULUS \\
& CTE
\end{tabular}

PREPREG DEVELOP.

TENSILE
COMP
SHEAR
MOUULUS

$$
\text { TAPE }
$$

ROSETTE

3-D ORTHOGONAL

3-D INTERLOCK

3-D INTREMOLD III

\section{STIFFENER DEVELOP.}

MACERATION
TEE BAR
HOINEYCOINB

LAYUP DEVELOP.

$\begin{array}{ll}\text { BWF } & \text { RT } \\ \text { BWF } & \text { RT } \\ \text { WF } & \text { RT } \\ \text { BWF } & \text { RT } \\ \text { BWF } & \text { RT }\end{array}$

RT
RT
RT
RT
RT

RT
RT
RT
RT

RT

RT
RT
RT

RT
RT
RT

OP
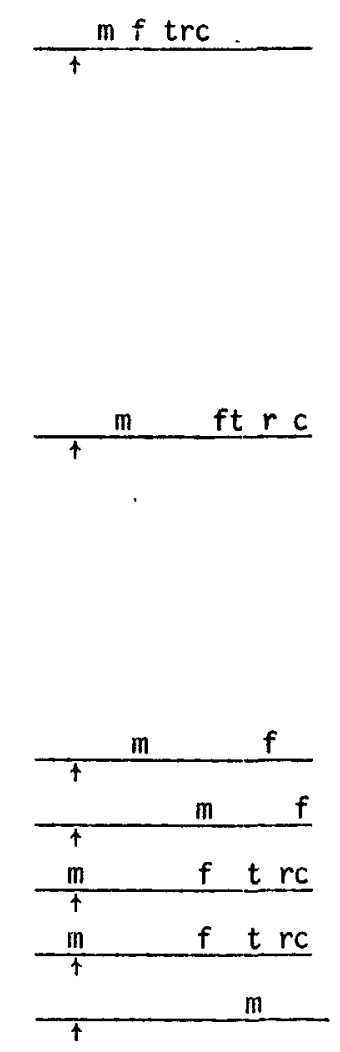

"Gross Flange Section Tests

*Gross Flange Section Tests 
M-6 FIBROUS GRAPHITE COHPOSITE MATERIALS DATA AND MATURITY STATUS (MATERIALS IRRADIATION)

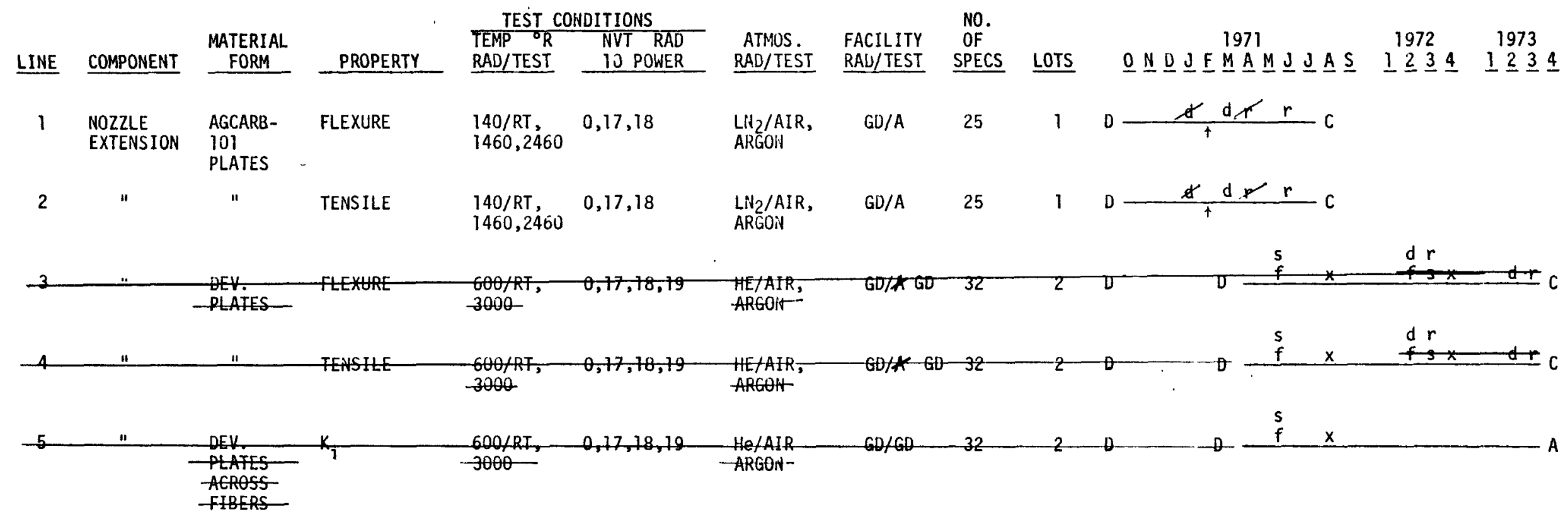


H-7 AA 6J6l-T6

MATERIAL PRIORITY ANU MATURITY STATUS
15 FEBRUARY 1971

(INVESTIGATOR: O. J. LAMVERMEYER)

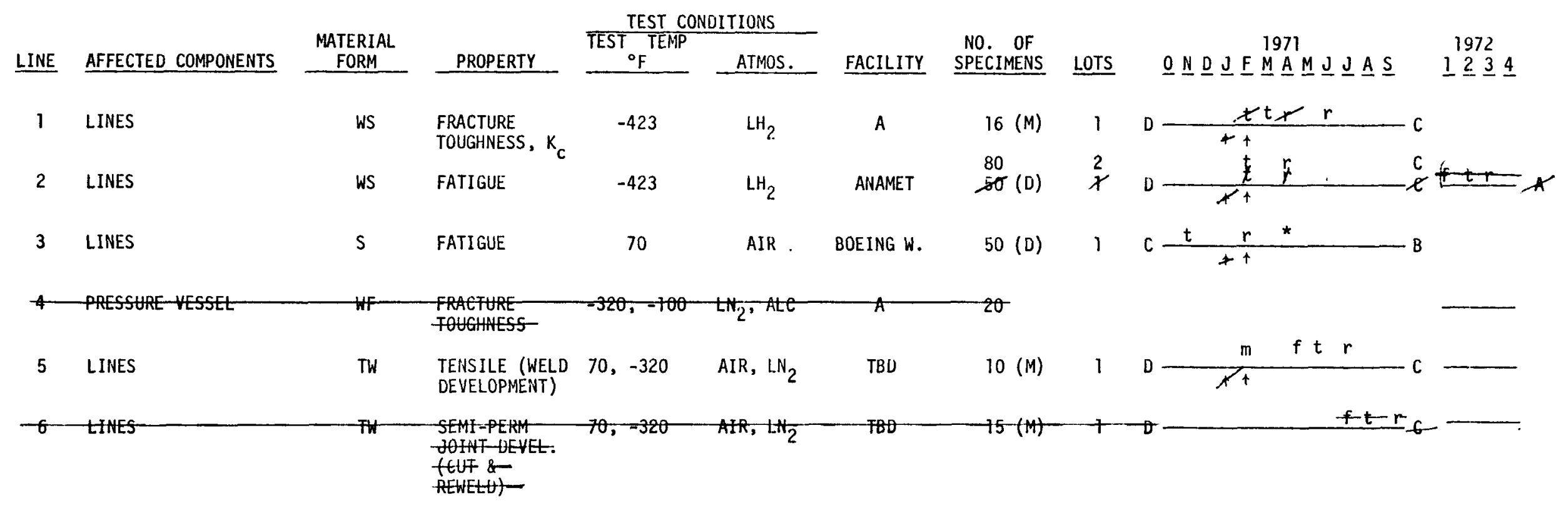

${ }^{\star}$ COMPLETE 1 FEBRUARY 1971. 
A. $\sim . M-7.6061-T 6$

MATERIALS DATA AND MATURITY STATUS (MATERIALS IRRAUIATION)
15 FEBRUARY 1971

(INVESTIGATORS: C. E. DIXON/ T. A. REDFIELO)

\begin{tabular}{|c|c|c|c|c|c|c|c|c|c|c|c|c|}
\hline & & & & TEST & ITIONS & & & & & & & \\
\hline LINE & COMPONENT & $\begin{array}{l}\text { MATERIAL } \\
\text { FORM } \\
\end{array}$ & PROPERTY & $\begin{array}{l}\text { TEMP }{ }^{\circ} R \\
\text { RAD/TEST }\end{array}$ & $\begin{array}{l}\text { NVT RAD } \\
10 \text { POWER }\end{array}$ & $\begin{array}{l}\text { ATMOS. } \\
\text { RAD/TEST }\end{array}$ & $\begin{array}{l}\text { FACILITY } \\
\text { RAD/TEST }\end{array}$ & $\begin{array}{l}\text { OF } \\
\text { SPECS }\end{array}$ & LOTS & ONDJF MAM J JAS & 1972 & $\begin{array}{l}1973 \\
1234\end{array}$ \\
\hline
\end{tabular}

1 PV HEAD 606I-T6

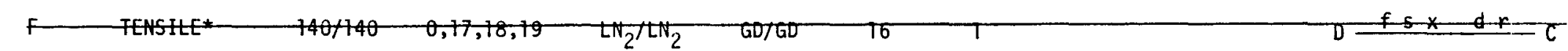

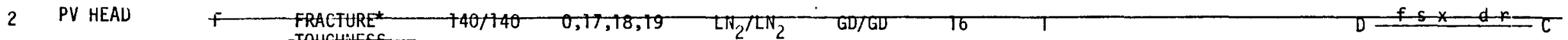

3 PV HEAD

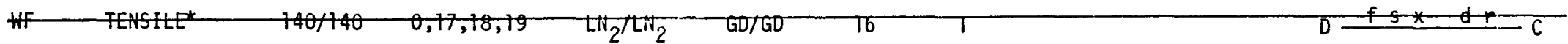

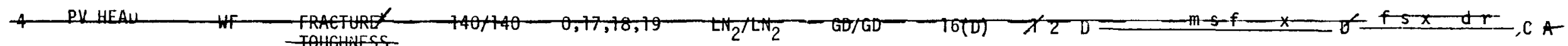

Tines

TEHSTEE

$40 / 40-0,18,19$

$\mathrm{LH}_{2} / \mathrm{LH}_{2}-\mathrm{GD} / \mathrm{G}$

12

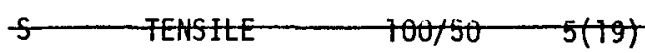
GHe/LIQ PT NEON

7 WIAES

77 $\mathrm{AH} / \mathrm{tH}-\mathrm{GU}-\mathrm{GU}$

$7 P-40$

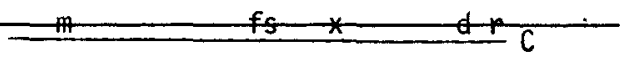

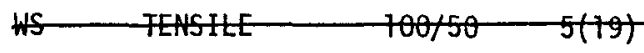
GHe/LIQ
NEON

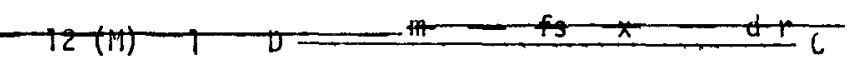




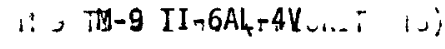

MATERIAL PRIORITY AND MATURITY STATUS
15 FEBRUARY. 1971

(INVESTIGATOR:

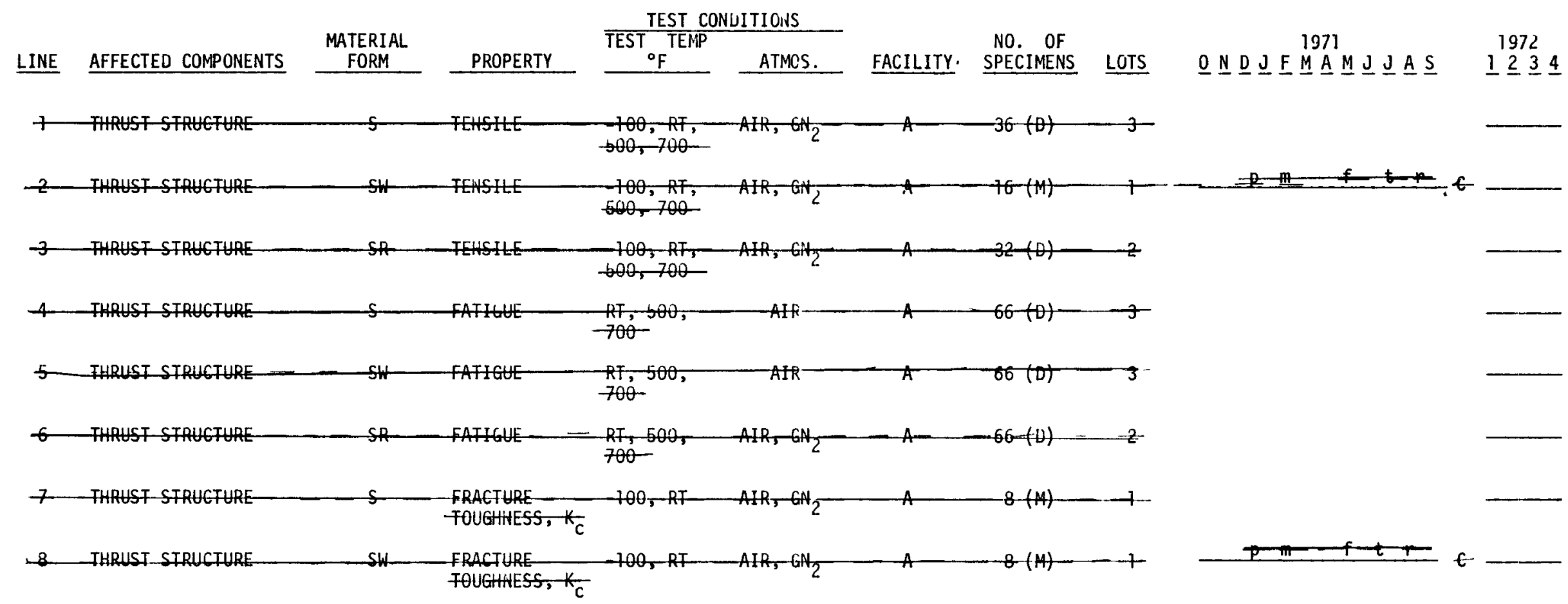


$M-9$ TI $-6 A L-4 V$

MATERIALS DATA AND MATURITY STATUS (MATERIALS IRRADIATION)
15 FEBRUARY 1971

is , , m

(INVESTIGATORS: C. E. UIXON/T. A. REUFIELD)

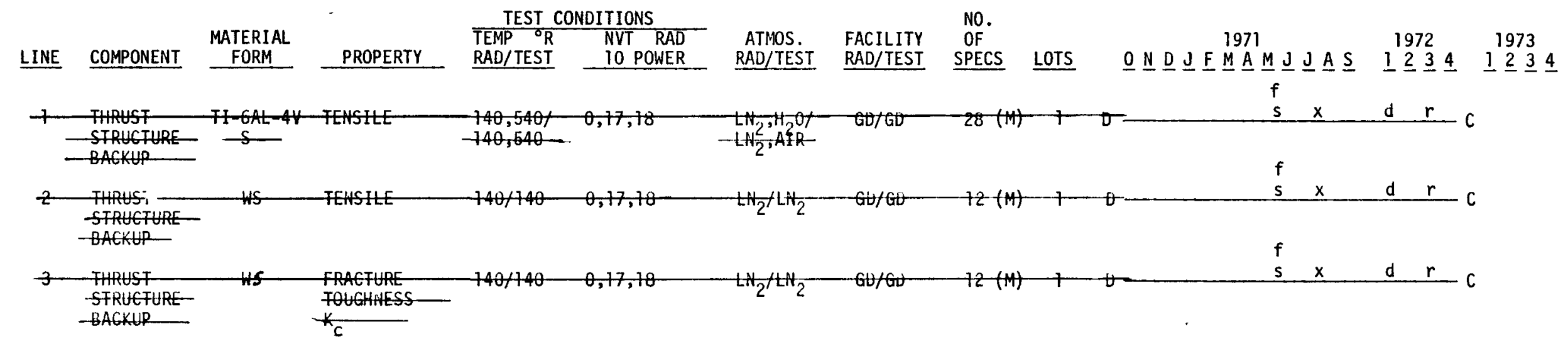


M-10 AISI 301 STAINLESS STEEL, $1 / 4$ HARD

LINE

MATERIAL

FORM
TEST CONDITIUNS PROPERTY

FACILITY

NO. OF

SPECIMENS

(NOMINAL)
15 FEBRUARY 1971

(INVESTIGATOR: U. J. LAMVERAEYER)
WALYES O ACTUATORS
TENSILE
$-425,-300$

RT
-AIR-
LOTS

$\underline{O} \underline{N} \underline{D} \underline{\mathrm{J}} \underline{F}^{1971} \underline{\mathrm{A}} \underline{M} \underline{\mathrm{J}} \underline{\mathrm{J}} \underline{\mathrm{A}} \underline{\mathrm{S}}$

$30(\mathrm{D})$ $2-\mathrm{c}-\mathrm{m} \quad \mathrm{r}$ 
M-11 FRICTION ANU WEAR

MATERIAL PRIORITY AND MATURITY STATUS
15. FEBRUARY 1971

(INVESTIGATOR: D. J. LAMVERMEYER)

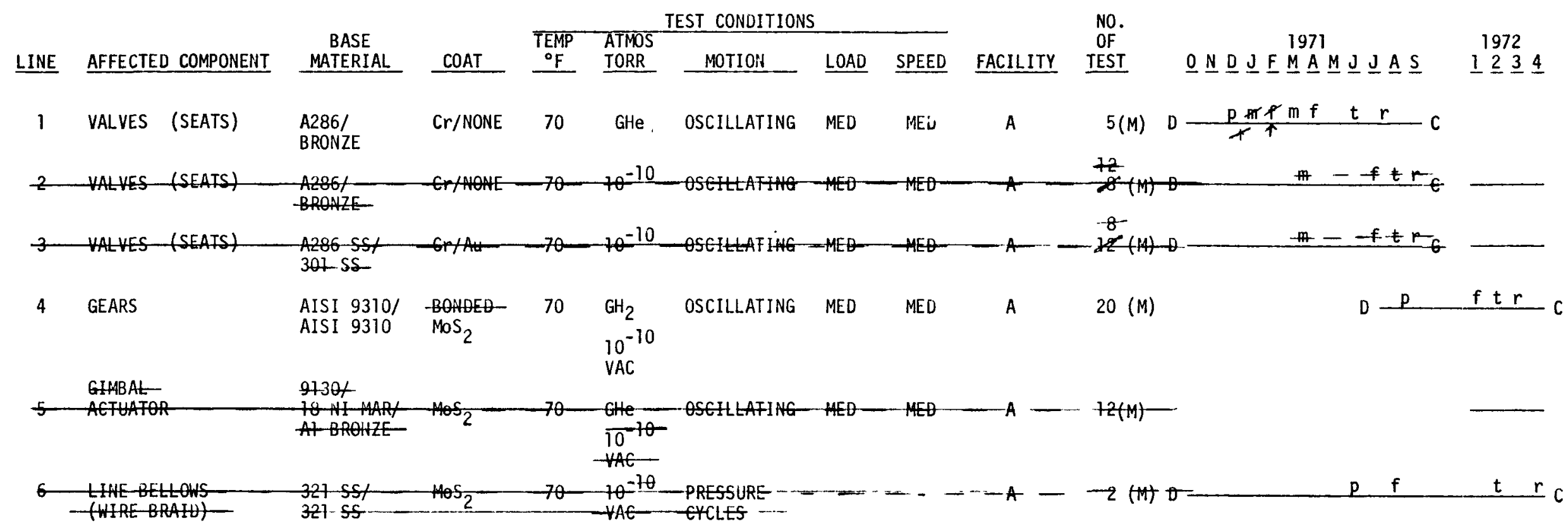


M-12 IIASTELLOY $X$

MATERIAL PRIORITY AND MATURITY STATUS
15 FEBRUARY 1971

(INVESTIGATOR: W. F. EMMONS)

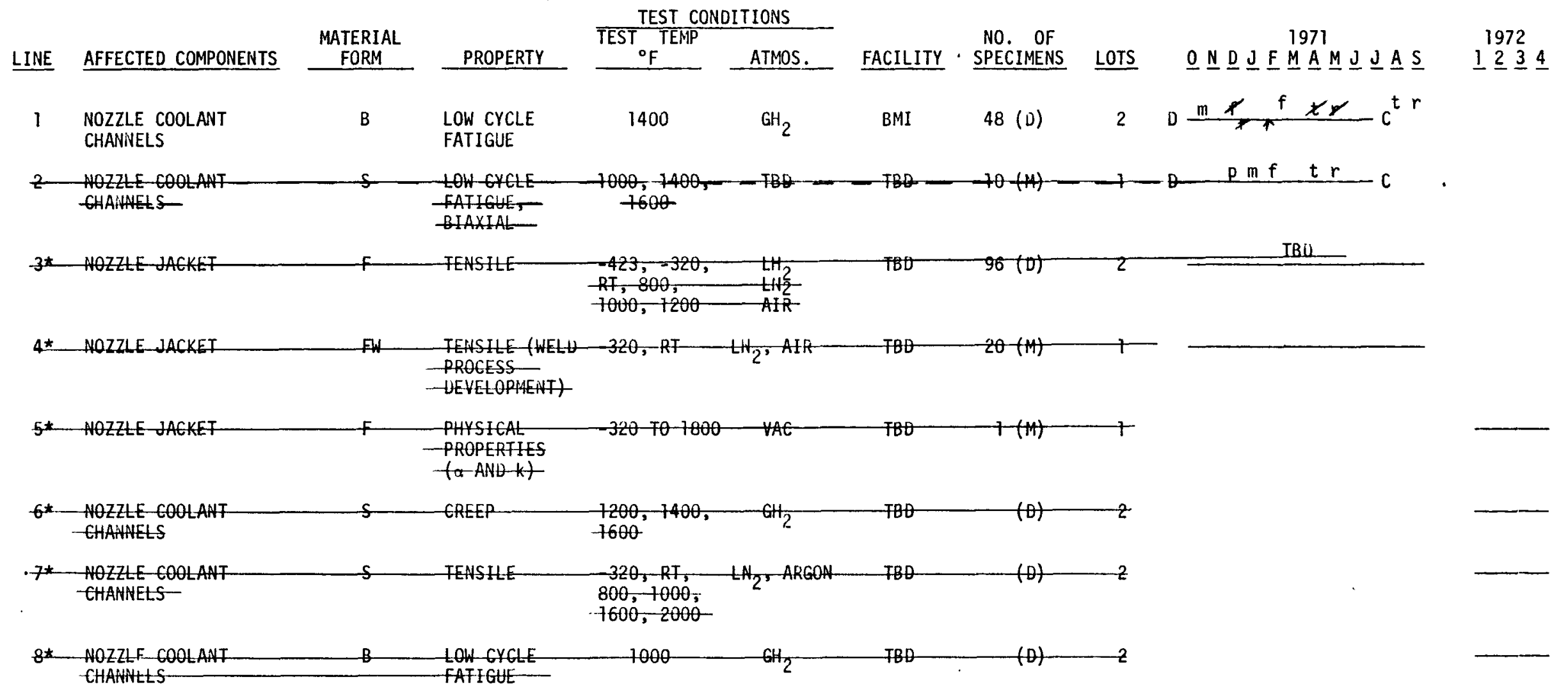

* THIS WORK WHLL HOT BE DONE IF ARMEO 22-73-5-IS SELLCFEU (SEE-M-38): 
H-12 HASTELLOY $X$

MATERIALS DATA AND MATURITY STATUS (MATERIALS IRRADIATION)
15 FEBRUARY 1971

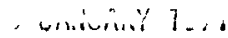

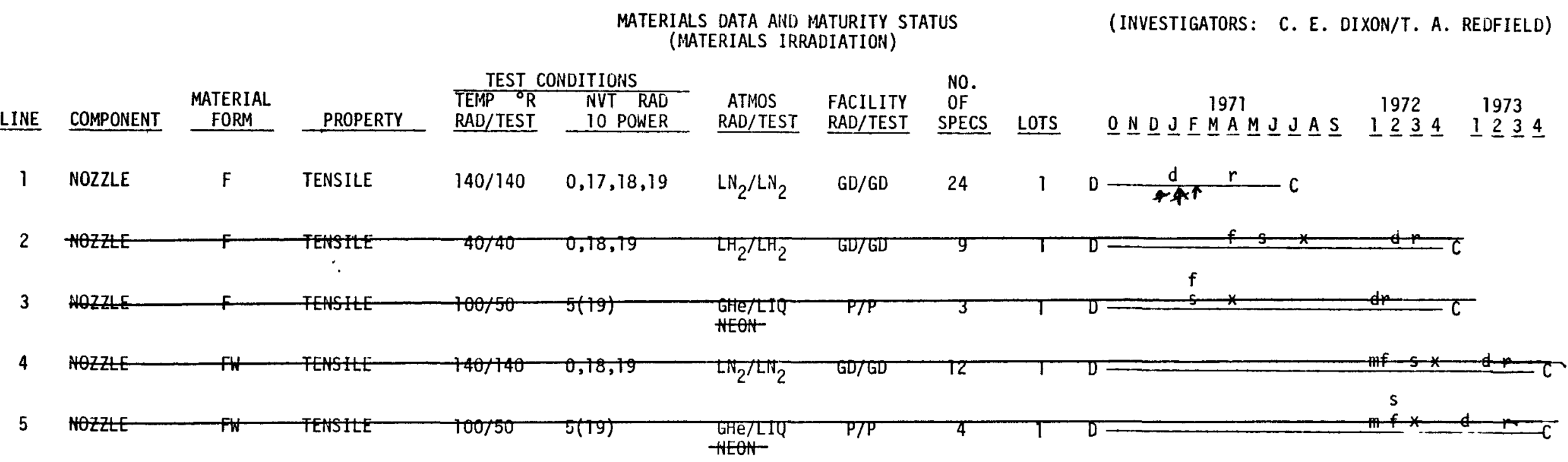


II-14 FEURALOH POLYIIIIUE

MATERIAL DATA AND MATURITY STATUS (MATERIALS IRRADIATION)
15 FEBRUARY 1971

\begin{tabular}{|c|c|c|c|c|c|c|c|c|c|c|c|c|c|c|}
\hline LINE & COMPONENT & $\begin{array}{c}\text { MATERIAL } \\
\text { FORM } \\
\end{array}$ & PROPERTY & $\begin{array}{r}\text { TEST } \\
\text { TEMP } \\
\text { RAD/TEST } \\
\end{array}$ & $\begin{array}{l}\text { ONDITIONS } \\
\gamma \text { RAD } \\
10 \text { POWER } \\
\end{array}$ & $\begin{array}{c}\text { ATMOS. } \\
\text { RAL/TEST }\end{array}$ & $\begin{array}{l}\text { FACILITY } \\
\text { RAU/TEST } \\
\end{array}$ & $\begin{array}{l}\text { No. } \\
\text { OF } \\
\text { SPECS } \\
\end{array}$ & LOTS & $\underline{0} \underline{N} \underline{\mathrm{J}} \underline{F}$ & $\begin{array}{l}1971 \\
M \underline{A} \underline{M} \underline{J}=\end{array}$ & $A \underline{S}$ & $\begin{array}{r}1972 \\
1 \leq 2\end{array}$ & $\begin{array}{c}1973 \\
1 \leq 3 \leq 4\end{array}$ \\
\hline 1 & $\begin{array}{l}\text { ACTUATOR } \\
\text { BEARING } \\
\text { CAGE }\end{array}$ & FEURALON & TENSILE & $\begin{array}{c}40 / 140 \\
540\end{array}$ & $0,10^{9} \mathrm{RAU} / \mathrm{SAC}$ & $\begin{array}{l}\mathrm{LH}_{2} / \mathrm{LN}_{2}, \\
\mathrm{AIR}\end{array}$ & $\stackrel{*}{\mathrm{GU} / \not} \mathrm{GD}$ & $16(0)$ & $\not \gamma 2$ & $\frac{m}{4-1}$ & $f_{m \times 15}$ & 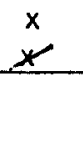 & $d r$ & $C A$ \\
\hline 2 & $"$ & " & FLEXURE & $\begin{array}{c}40 / 140 \\
540\end{array}$ & $0,10^{9} \mathrm{RAD} / \mathrm{GM}$ & $\begin{array}{l}\mathrm{LH}_{2} / \mathrm{LN}_{2} \\
\text { AIR }\end{array}$ & $\stackrel{\star}{\mathrm{GD} / \not K \mathrm{GU}}$ & $\begin{array}{l}16(0) \\
12\end{array}$ & $\gamma 2$ & $\frac{m}{4}$ & f, $n x \times f s$ & $\stackrel{x}{x}$ & $d r$ & A \\
\hline
\end{tabular}

*IN CONJUNCTION WITH GTR-MFSC SCHEDULEU FOR 9 MAY. MAY NOT NEEU $10^{9}$ RAU. 
.. M-T6 MARAGING STEEL (18 NI)

MATERIAL PRIORITY AND MATURITY STATUS
15 FEBRUARY 1971

(INVESTIGATOR: W. F. EMMONS)

\begin{tabular}{|c|c|c|c|c|c|c|c|c|}
\hline \multirow[b]{2}{*}{ LINE } & \multirow[b]{2}{*}{ AFFECTED COMPONENTS } & \multirow[b]{2}{*}{$\begin{array}{c}\text { MATERIAL } \\
\text { FORM } \\
\end{array}$} & \multicolumn{3}{|c|}{ TEST CONUITIONS } & \multirow[b]{2}{*}{ FACILITY. } & \multirow[b]{2}{*}{$\begin{array}{l}\text { NO. OF } \\
\text { SPECIMENS }\end{array}$} & \multirow[b]{2}{*}{ LOTS } \\
\hline & & & PROPERTY & $\begin{array}{l}\text { TEST TEIIP } \\
{ }^{\circ} \mathrm{F}\end{array}$ & ATMOS. & & & \\
\hline 1 & $\begin{array}{l}\text { GIMBAL FLEXURE } \\
\text { PIVOT }\end{array}$ & $P-B$ & TENSILE & $\begin{array}{l}-320, \mathrm{RT}, \\
300\end{array}$ & $\mathrm{LN}_{2}, \mathrm{AIR}$ & A & $24(D)$ & 2 \\
\hline 2 & Q AMBAL & PW $B W$ & TENSHE & $300,-R T$ & $t+A, A L$ & 4 & $-24-(0)$ & 2 \\
\hline-3 & GIMAL & $+\bar{B}$ & FATIGUE- & $-300=A T$ & AIR & $-A$ & $-6 \theta(\theta)$ & -2 \\
\hline-4 & GIMABAL & $P H=$ & FATIGUE & $-3 Z=R T$ & $-\mathrm{N}_{2}$ & $-A$ & $-60(0)$ & -2 \\
\hline 5 & GIMBAL & $P \quad B$ & $\begin{array}{l}\text { FRACTURE } \\
\text { TOUGHNESS, }\end{array}$ & $K_{I C}-320, R T$ & $L N_{2}, A I R$ & $A$ & $\begin{array}{l}24(D) \\
+2(M)\end{array}$ & 2 \\
\hline 6 & GIMBAL & $P \quad B$ & $\begin{array}{l}\text { STRESS } \\
\text { CORROSION }\end{array}$ & RT & TBU & A & $90(M) *$ & 1 \\
\hline-7 & GIMBAL & D $B$ & $\begin{array}{l}\text { CORROSLOH } \\
\text { FATIGUE }\end{array}$ & -RF & $-T B Q$ & $-A$ & $-48+4)^{k}$ & 7 \\
\hline
\end{tabular}
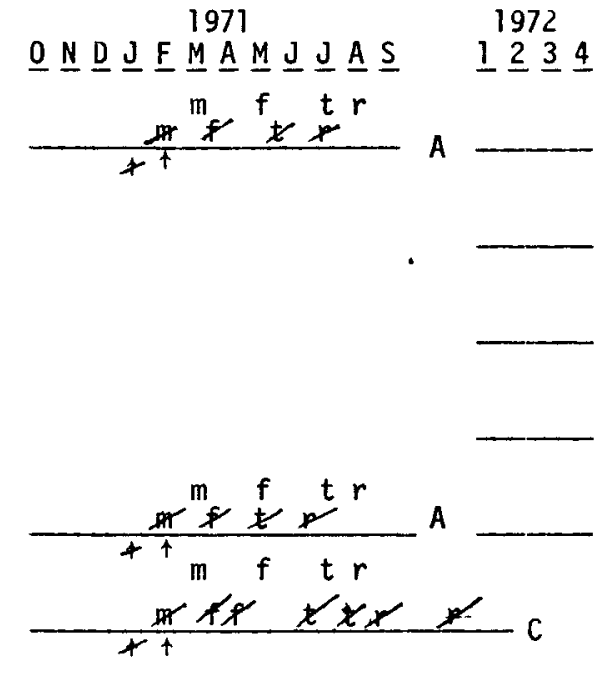

$\star / 3$ COATINGS 
M-16 18 ilI IARAGING STEEL

MATEKIALS UATA ANU MATURITY STATUS

(MATERIALS IRRAUIATION)
15 FEBRUARY 1971

(INVESTIGATORS: C. E. DIXON/T. A. REUFIELD)

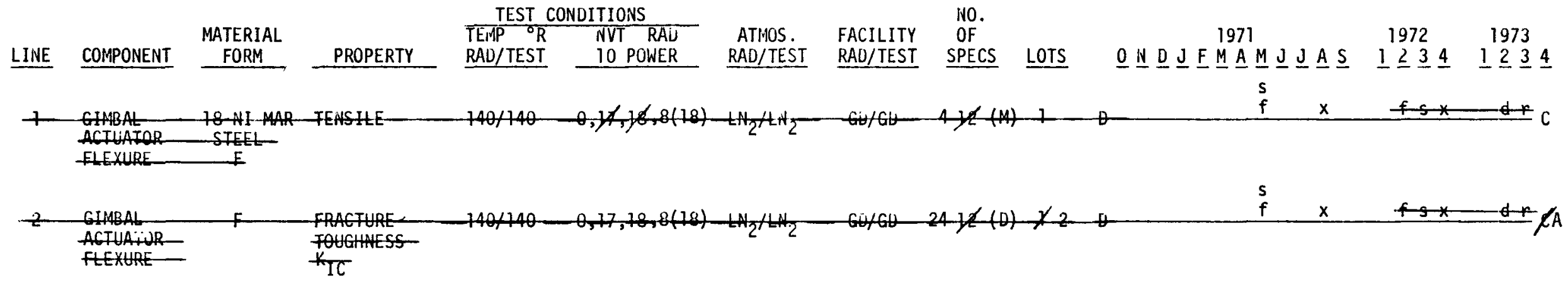


M-19 AISI .440C

MATERIAL PRIORITY AND MATURITY STATUS
15 FEBRUARY 1971

(INVESTIGATOR: P. P. LESSAU)

\begin{tabular}{|c|c|c|c|c|c|c|c|c|}
\hline \multirow[b]{2}{*}{ LINE } & \multirow[b]{2}{*}{ AFFECTED COMPONENTS } & \multirow[b]{2}{*}{$\begin{array}{l}\text { MATERIAL } \\
\text { FORIM } \\
\end{array}$} & \multirow[b]{2}{*}{ PROPERTY } & \multicolumn{2}{|c|}{ TEST CONDITIONS } & \multirow[b]{2}{*}{ FACILITY } & \multirow[b]{2}{*}{$\begin{array}{l}\text { NO. OF } \\
\text { SPECIMENS }\end{array}$} & \multirow[b]{2}{*}{ LOTS } \\
\hline & & & & $\begin{array}{l}\text { TEST TEMP } \\
{ }^{\circ} \mathrm{F} \\
\end{array}$ & ATMOS. & & & \\
\hline 1 & TPA BEARINGS & B & HARDIVESS & $R T,-320$ & AIR, Lid $_{2}$ & A & $16(D)$ & 2 \\
\hline 2 & TPA BEARINGS & B & $\begin{array}{l}\text { MODULUS OF } \\
\text { ELASTICITY }\end{array}$ & RT, -423 & AIR, $\mathrm{LH}_{2}$ & A & $16(D)$ & 2 \\
\hline 3 & TPA BEARINGS & B & $\begin{array}{l}\text { POISSON'S } \\
\text { RATIO }\end{array}$ & $R T,-423$ & AIR, $\mathrm{LH}_{2}$ & A & $16(D)$ & 2 \\
\hline 4 & TPA BEARINGS & B & $\begin{array}{l}\text { COMPRESSIVE } \\
\text { YIELD }\end{array}$ & $R T,-423$ & AIR, $\mathrm{LH}_{2}$ & A & 16 (D) & 2 \\
\hline
\end{tabular}

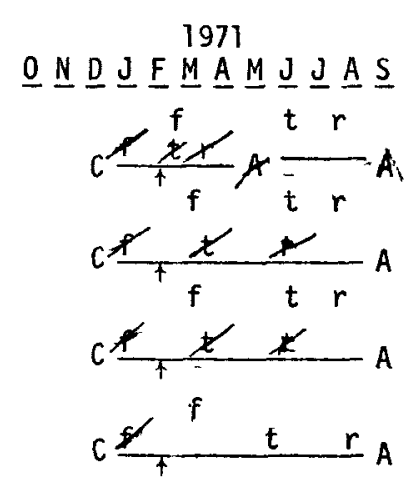

1972

$12 \underline{3} 4$ 
CURRENTLY NOT BEING CONSIDERED FOR LINES.

M-20 AA 5086

MATERIAL PRIORITY AND MATURITY STATUS
15 FEBRUARY. 1971

(INVESTIGATOR: W. F. EMMONS)

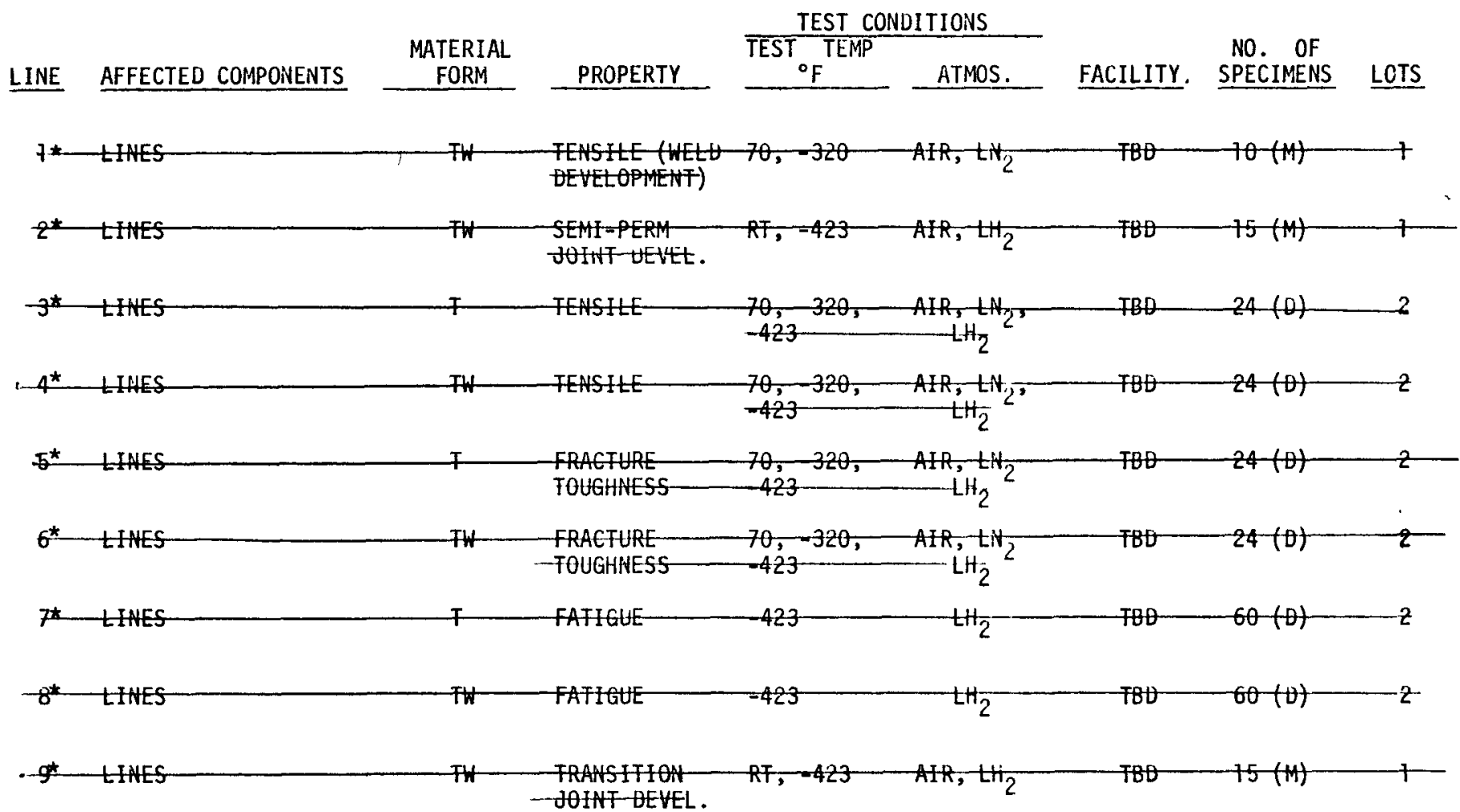

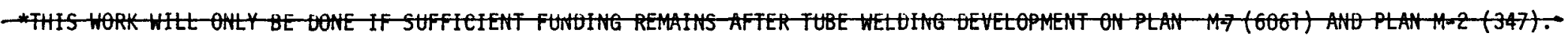


PAGE 20a

$M-203030$

MATERIALS DATA AND MATURITY STATUS (MATERIALS IRRAUIATION)
15 FEBRUARY 1971

(IIVVESTIGATORS: C. E. DIXON/T. A. REDFIELD)

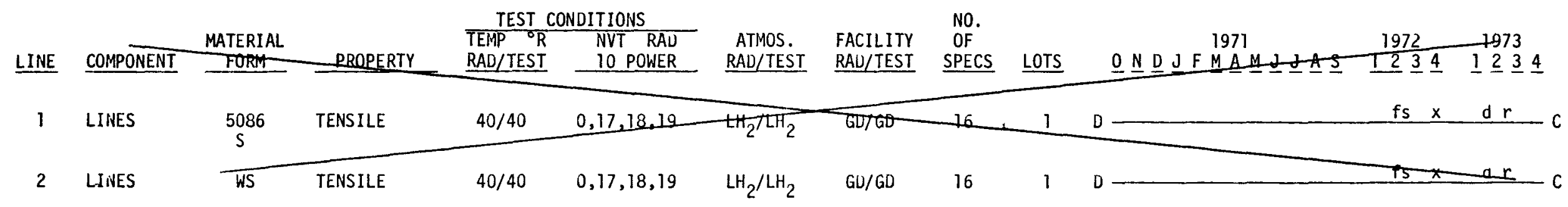


11-21 7075-T73

MATERIAL PRIORITY AND MATURITY STATUS
15 FEBRUARY 1971

(INVESTIGATOR: D. J. LAMVERMEYER)

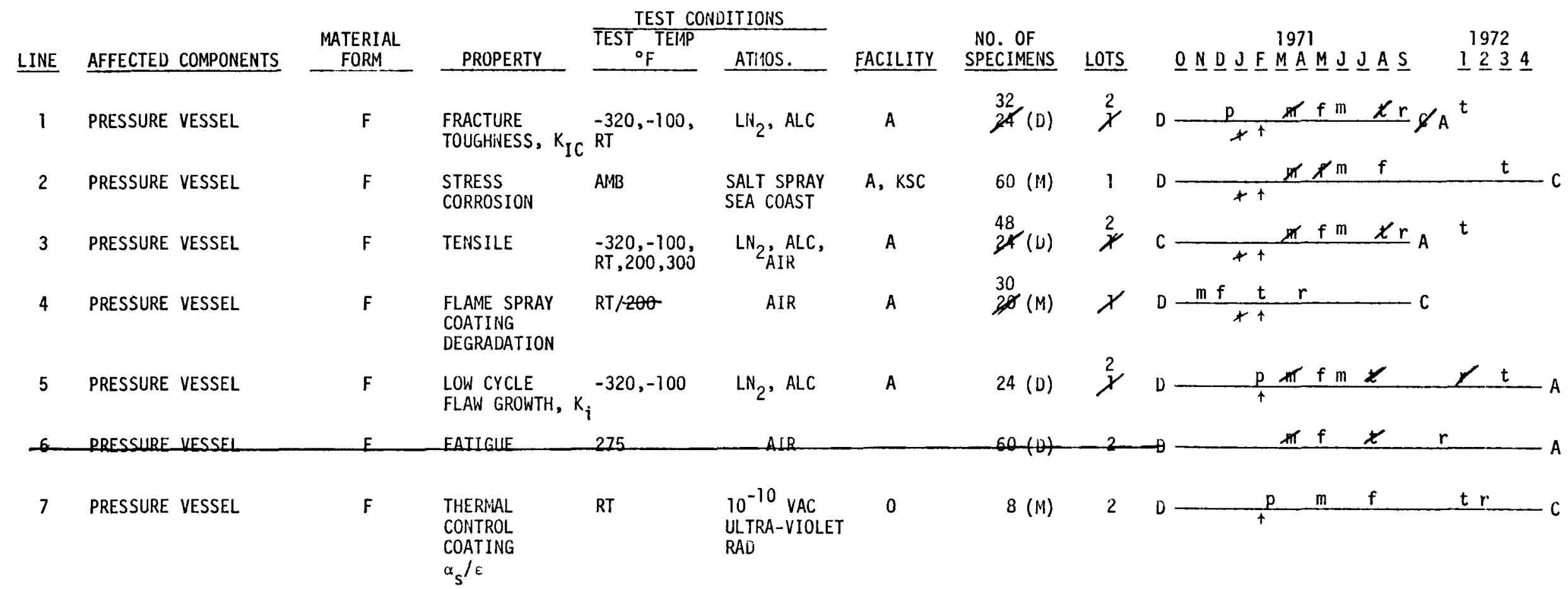


[1-2] AA 7075-T73

MATERIALS DATA AND MATURITY STATUS

(MATERIALS IRRAUIATION)
15 FEBRUARY 1971

(INVESTIGATORS: C. E. DIXON/T. A. REDFIELD)

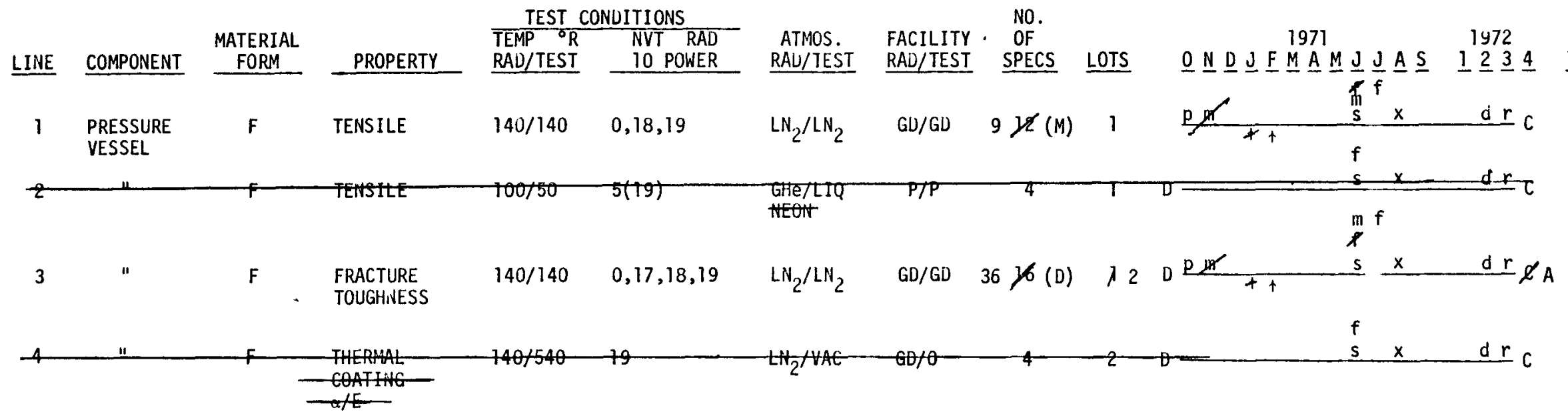


$M-22$ Gll; CABRITTLEIEIT

MATERIAL PRIORITY ANO MATURITY STATUS
15 FEBRUARY, 1971

(INVESTIGATOR: P. P. DESSAU)
LINE AFFECTED COMPONENTS

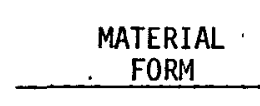

PROPERTY

TEST TEMP

TEST TEMP - ATMOS.

No. OF

SPECS.
LOTS $\quad \underline{0} \underline{N} \underline{D} \underline{J} \underline{F} \underline{M} \underline{M} \underline{M} \underline{\mathrm{J}} \underline{\mathrm{J}} \underline{A} \underline{S}$

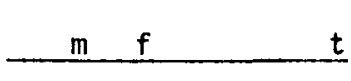

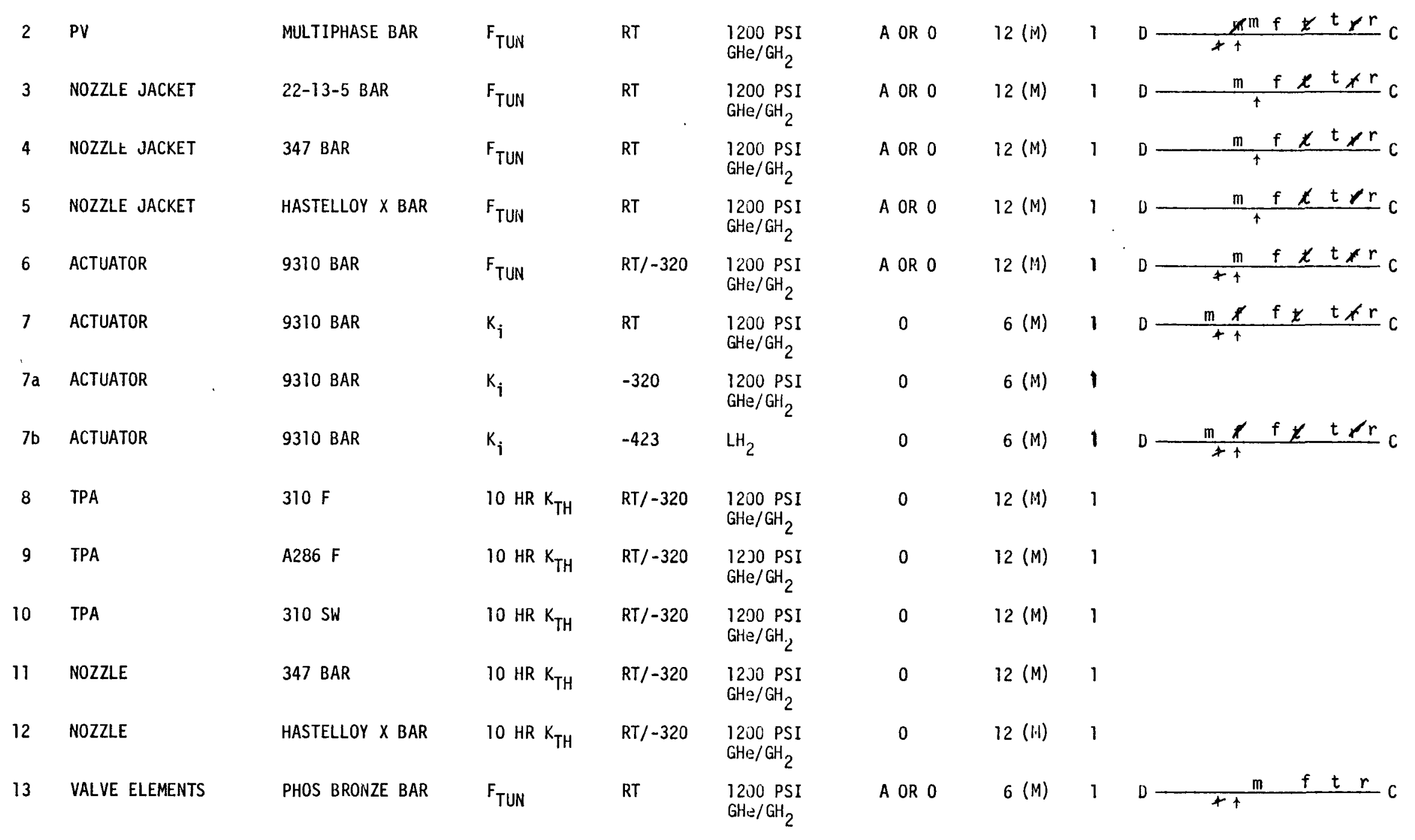


M-23 STRESS ANU RAUIATION SYNERGISTIC EFFECTS

MATERIALS DATA AND MATURITY STATUS (MATERIALS IRRADIATION)

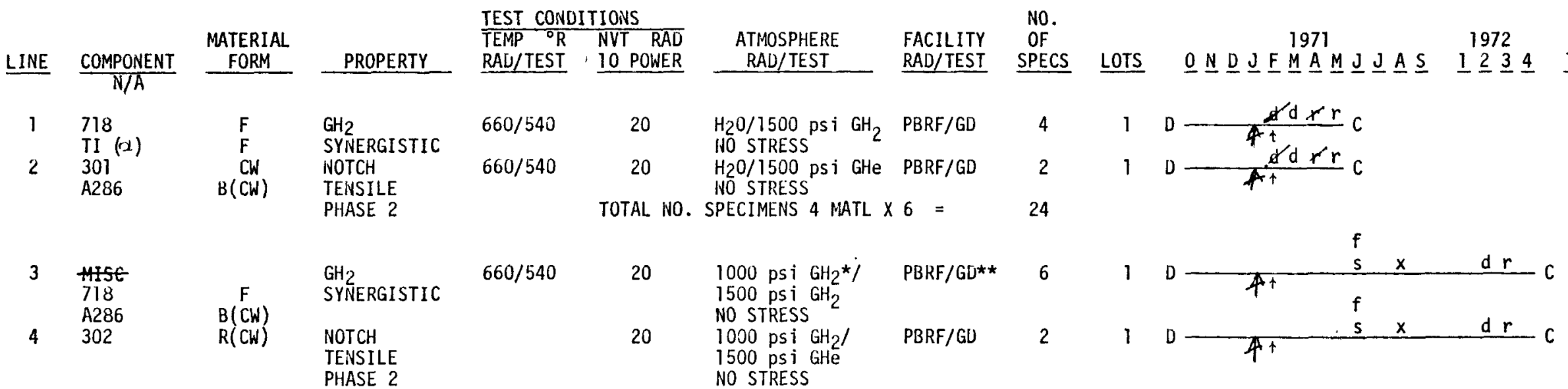

TOTAL NO. SPECIMENS 3 IHATL $\times 8=24$

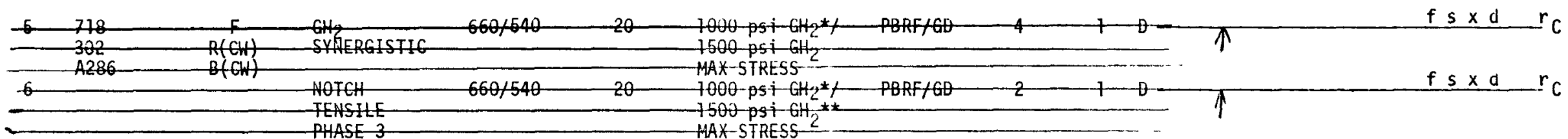

TOTAL NO. SPECIMENS 3 IMATLS $\times 6=18$

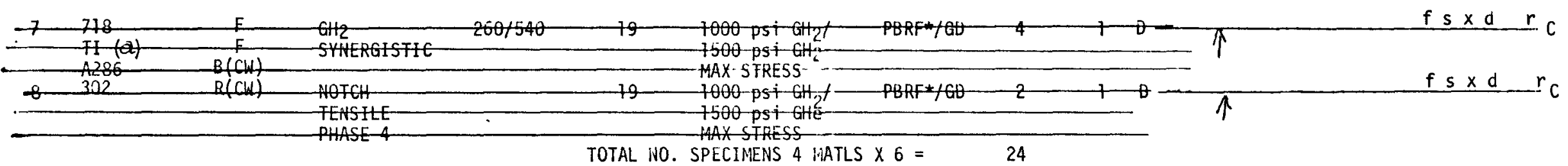

*GAGE SECTIONS OF SPECIMENS ARE ENCAPSULATED IN BELLOWS ANO PRESSURIZEO TO 1000 PSI (AT $200^{\circ} \mathrm{F}$ ).

**IRRADIATED SPECIMENS SHIPPED TO GU IN CAPSULE; CAPSULE PUNCTURED IN HIGH PRESSURE CLEAN GASEOUS HYDROGEN AND TESTED FOR NTS.

THESE RESULTS ARE COMPARED WITH AN IRRADIATION ATMOSPHERE OF 660 R REACTOR WATER. 


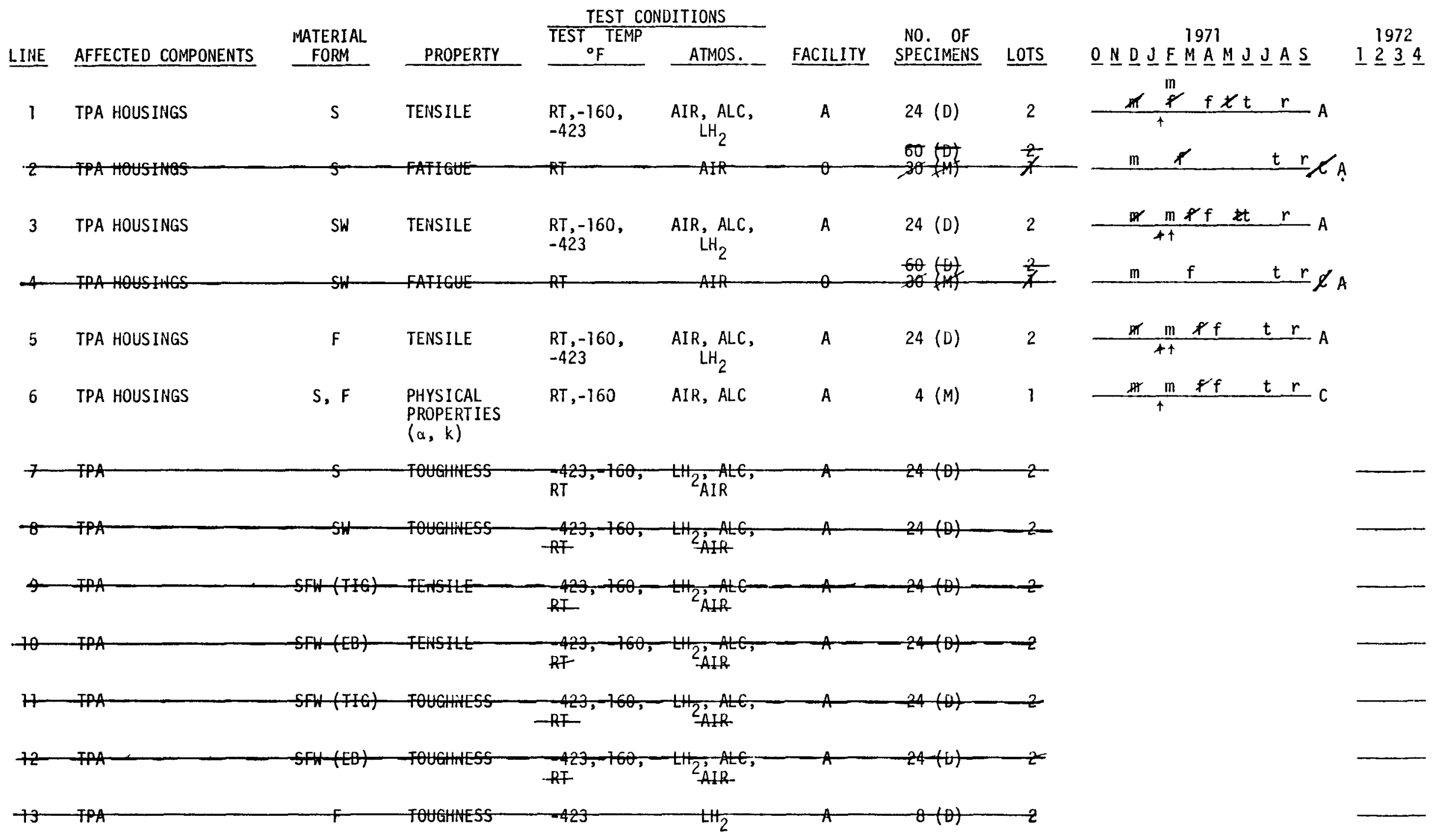


M-30 MULTIPHASE

MATERIAL PRIORITY AND MATURITY STATUS
15 FEBRUARY 1971

(INVESTIGATOR: D. J. LAMVERMEYER)

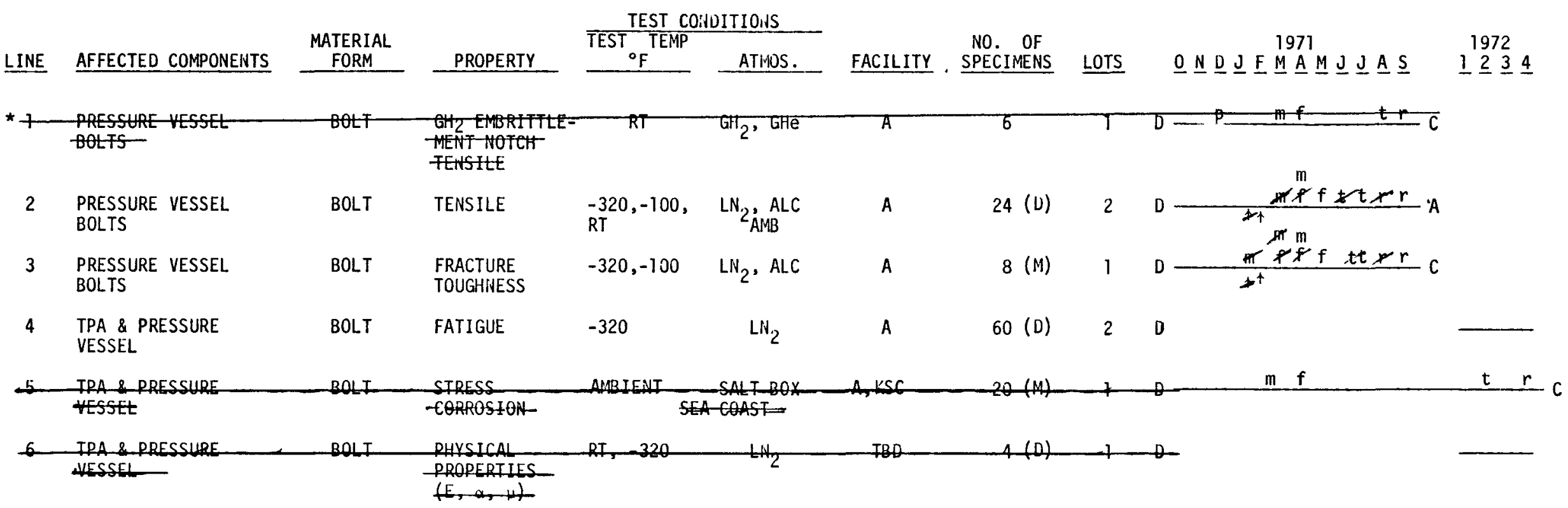

*ITEM TRANSFERRED TO TEST PLAN M-22. 
M-30 MULTIPHASE*

MATERIALS DATA AND MATURITY STATUS (MATERIALS IRRADIATION)
I5 FEBRUARY 1971

(INVESIIGATORS: C. E. DIXON/T. A. REDFIELD)

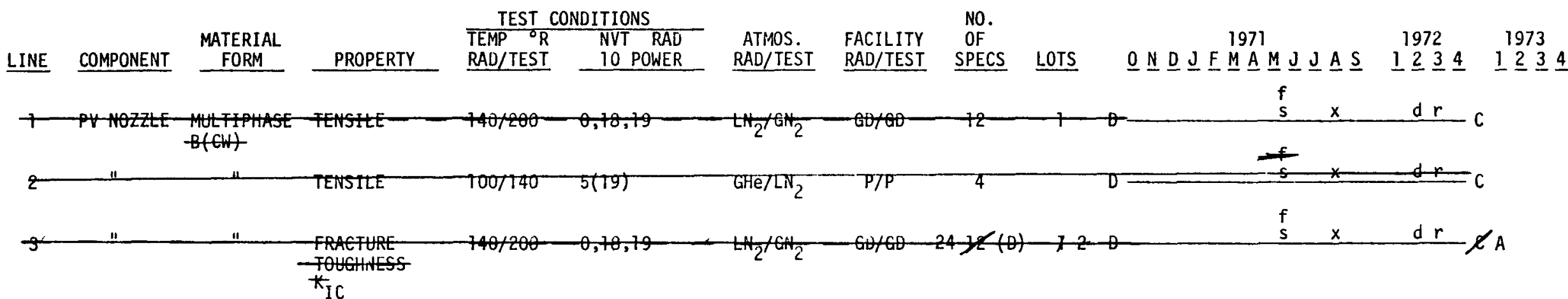

*IF THIS MATERIAL IS SUBJECT TO GH 2 EMBRITTLEMENT, IRRADIATION TEST WILL BE CANCELLED. 
$M-319310$

MATERIAL PRIORITY AND MATURITY STATUS
15 FEBRUARY 1971

(INVESTIGATOR: D. J. LAMVERMEYER)

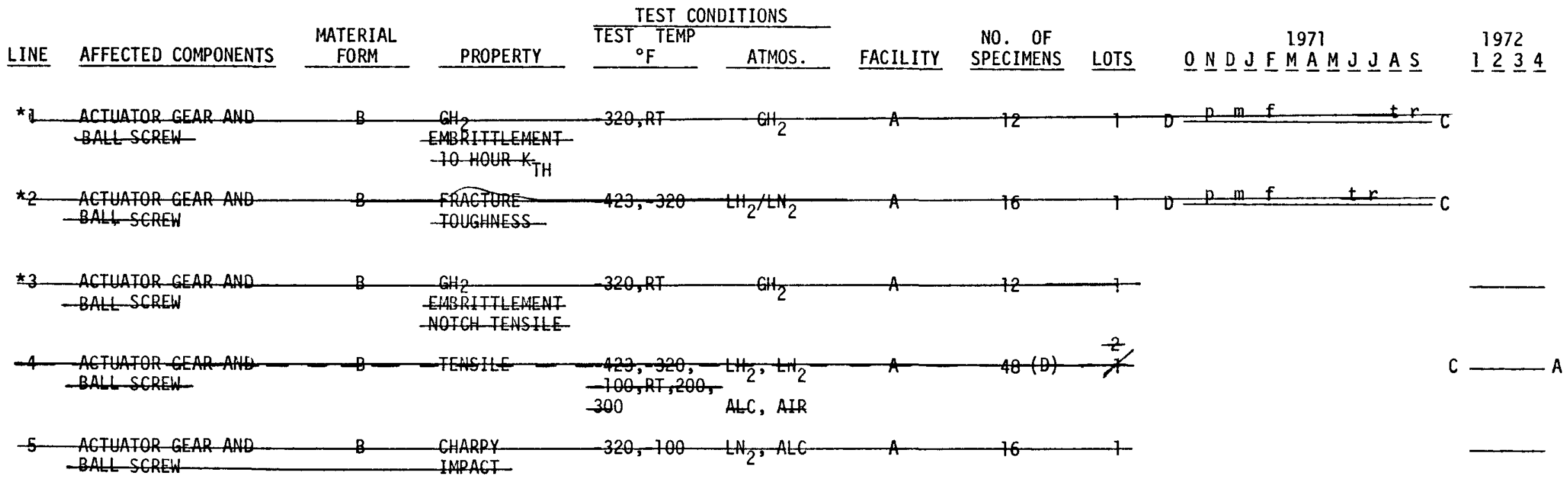

*ITEM TRANSFERRED TO TEST PLAN M-22. 
M-31 AISI 9310 LOW ALLOY STEEL

MATERIALS DATA AND MATURITY STATUS (IMATERIALS IRRADIATION)
15 FEBRUARY 1971

(INVESTIGATORS: C. E. DIXON/T. A. RELFIELD)

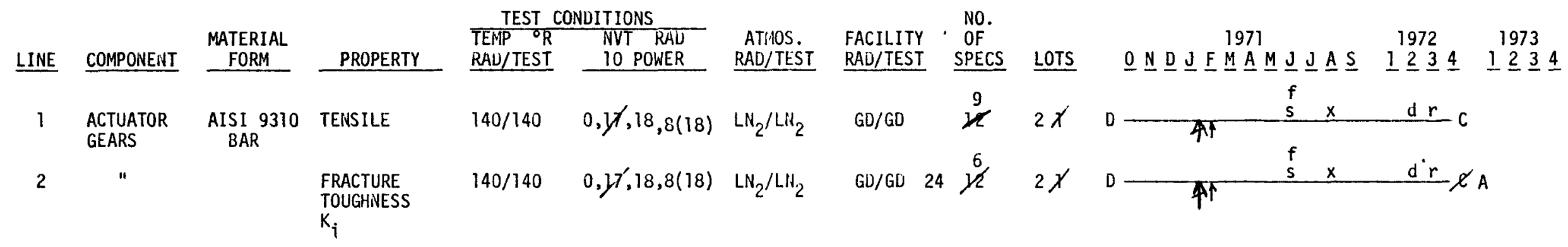


M-37 BRONZE

MATERIAL PRIORITY AND MATURITY STATUS
15 FEBRUARY 1971

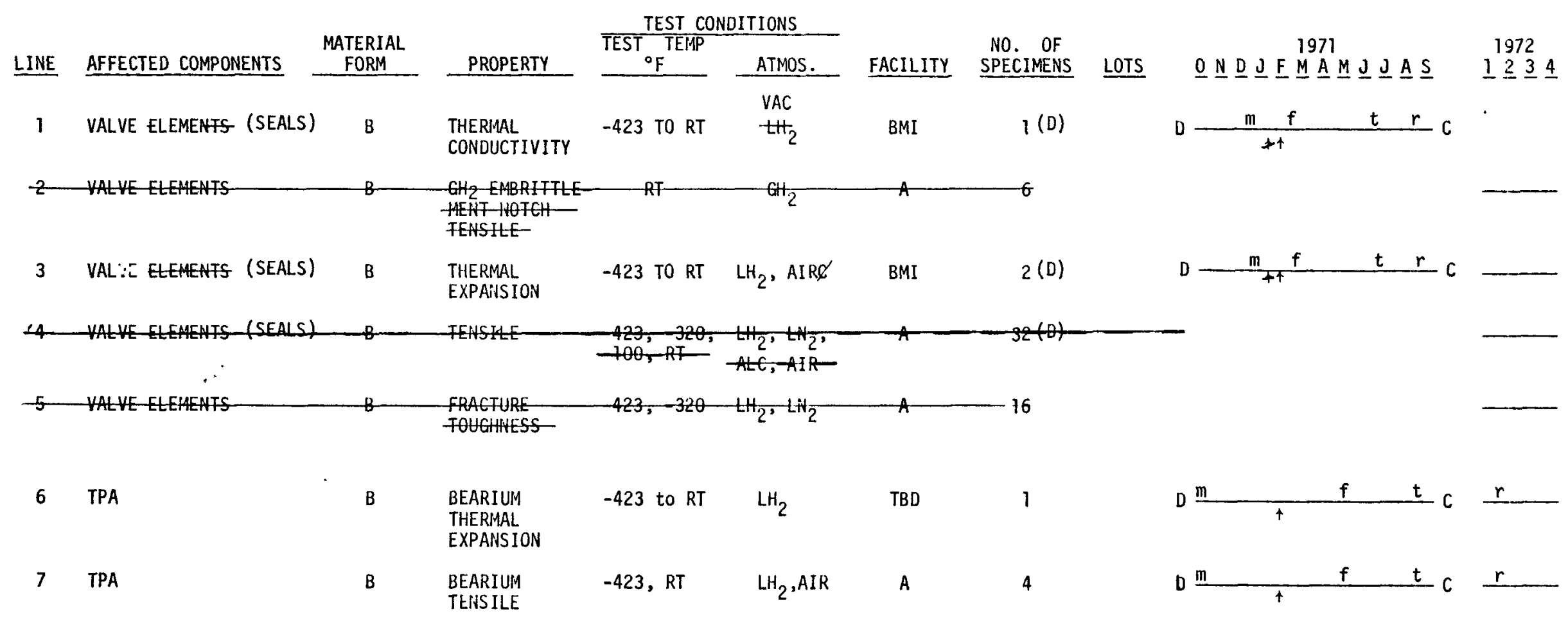




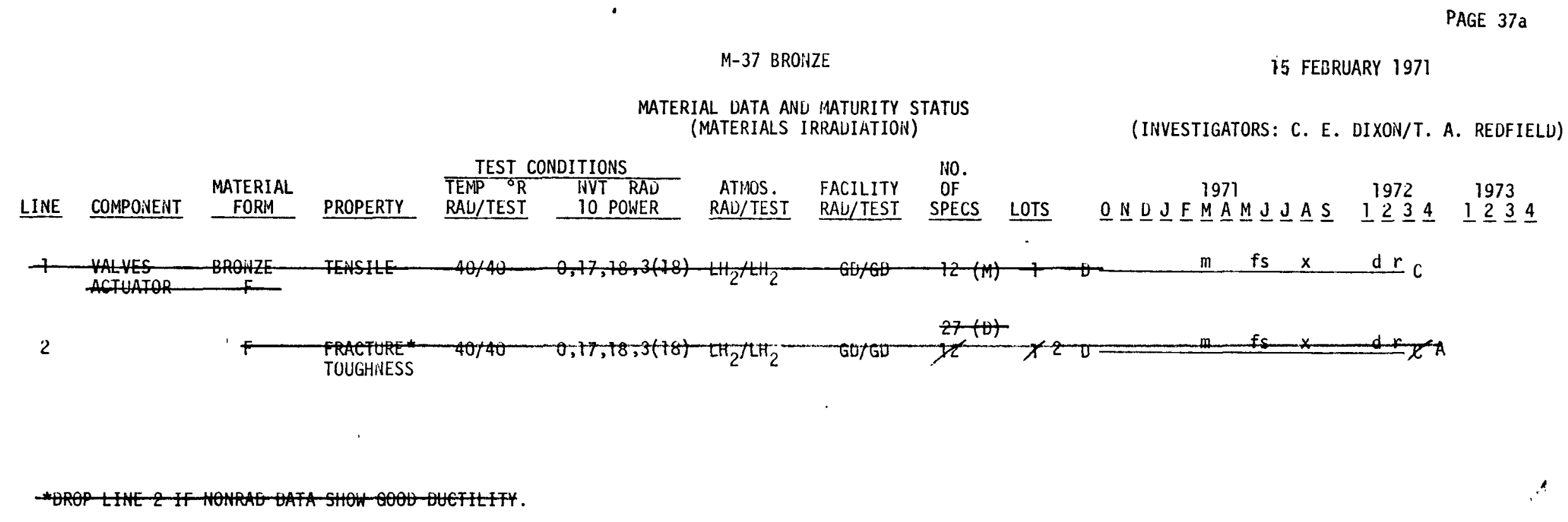




\section{M-38 STAINLESS STLEL 22-13-5 (ARMCO)}

MATERIAL PRIORITY AND MATURITY STATUS
15 FEBRUARY 1971

(INVESTIGATOR: W. F. EMMONS)

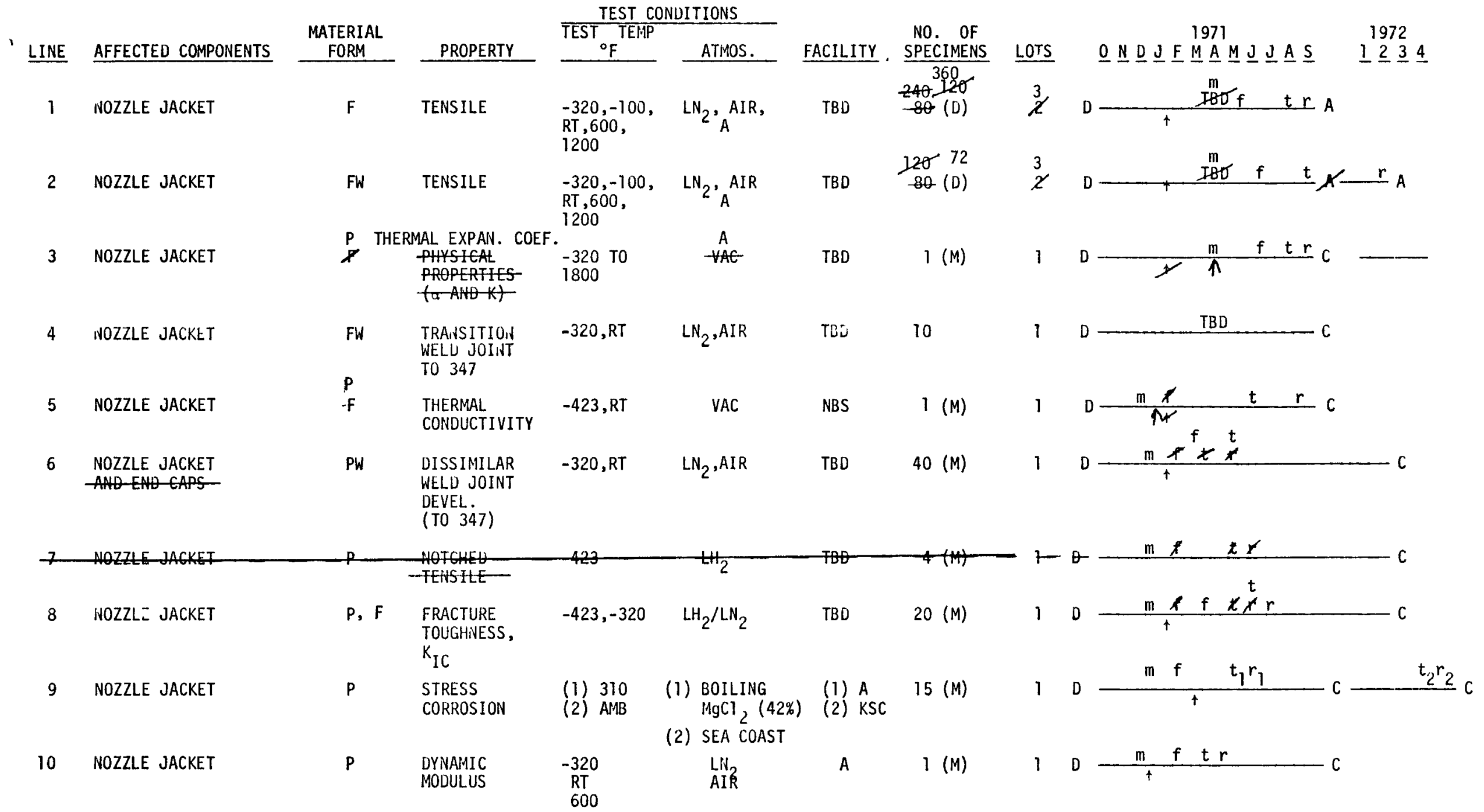


M-38 ARMICO ALLOY 22-13-5

MATERIAL DATA AND MATURITY STATUS (MATERIALS IRRAUIATION)

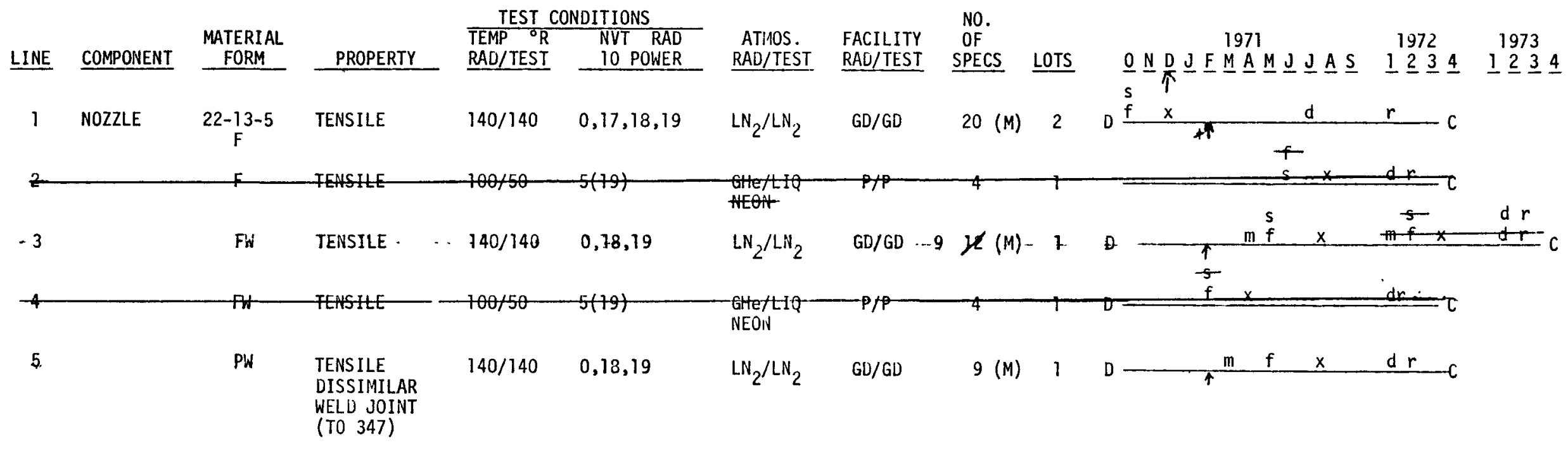


11-39 BeCu

MATERIAL PRIORITY AND MATURITY STATUS
15 FEBRUARY 1971

(INVESTIGATOR: D. J. LAMVERMEYER)

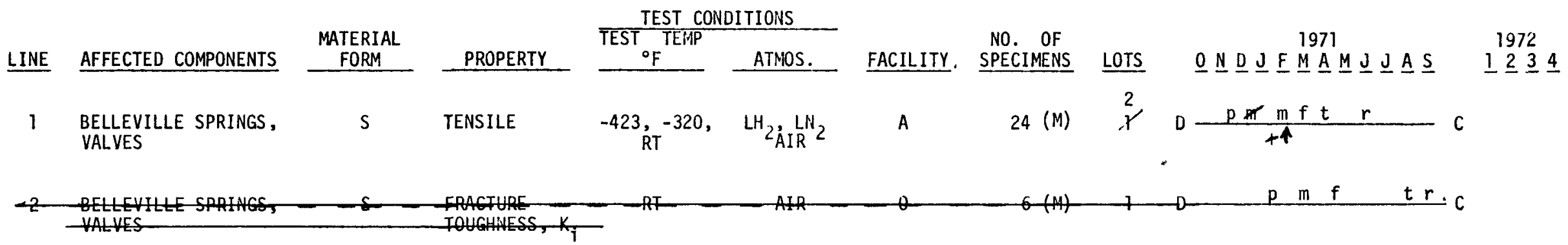


M-39 BeCu

MATERIAL PRIORITY AND MATURITY STATUS (MATERIALS IRRAUIATION)

$\therefore \quad 15$ FEBRUARY, 1971

INVESTIGATOR: C. E. DIXON

TEST CONDITIONS

AFFECTED MATERIAL

COMPONENTS FORM

PROPERTY ' TEMP ${ }^{\circ} \mathrm{R}$

ATMOS. FACILITY

RAD/TEST RAD/TEST

NO. OF

SPECS

LOTS

$\mathrm{LH}_{2} / \mathrm{LH}_{2}$

GD/GD

$4(M)$

O N D J F M A M J J A S $\quad 123$

$40 / 40$

0,18

GD/GD

$0,17,18$

$\mathrm{LH}_{2} / \mathrm{LH}_{2}$

TOUGHNESS,

$40 / 40$

SPRINGS 
MATERIALS DATA ANU MATURITY STATUS $i$ (MATERIALS IRRAUIATION)

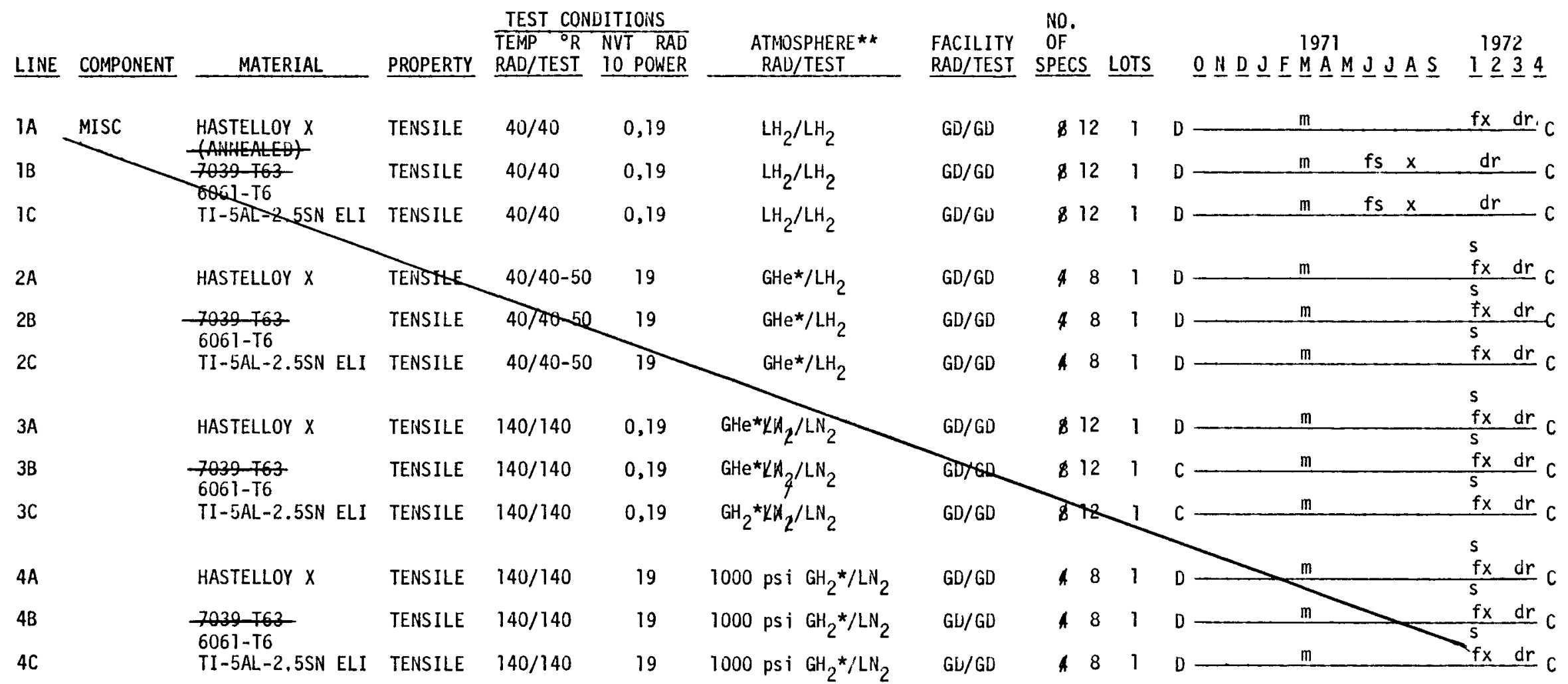

LINES 3B and 3C ARE DUPLICATES OF GTR-20C IN THE EVENT DIFFERENT HEATS ARE USEI).

\section{^ENCAPSUlated.}

* HEAT TRANSFER CALCULATIONS REQUIRED PRIOR TO FINAL DECISION ON SPECIMENS TO BE ENCAPSULATED. ANSC REQUESTED BY SNPO-C TO TRY TO ACCELERATE LINES 2 THROUGH 4. 
M-44 AA 2219-T6

MATERIAL PRIORITY ANU MATURITY STATUS
15 FEBRUARY 1,971

(INVESTIGATOR: W. F. EMMONS) $\frac{\text { TEST CONDITIONS }}{\text { TEST }}$

MATERIAL

FORM

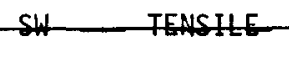

PROPERTY

ATMOS. FACILITY

150, RI, ALE, A $-200,300$ A
NO. OF

SPECS, LOTS
$\underline{O} \underline{D} \underline{J} \underline{F} \underline{M} \underline{A} \underline{M} \underline{\mathrm{J}} \underline{\mathrm{J}} \underline{A} \underline{S}$
1972

1 THRUST STRUGTURE

2 THRUST STRUGTURE 